\title{
The Dynamical Behaviour near Solitary Waves in Hamiltonian Lattice Differential Equations
}

\author{
Marc Georgi
}

\begin{abstract}
In this article we consider Hamiltonian lattice differential equations and investigate the existence of travelling waves near solitary wave solutions. We focus on the case where the solitary wave profile induces a homoclinic solution of the associated traveling wave equation, which is a typical scenario in the Fermi-Pasta-Ulam lattice. We will then show the existence of finitely many scalar bifurcation equations, such that zeros of these equations correspond to multi-pulses or periodic traveling waves of the original lattice equation that are located near the primary solitary wave. Compared to previous works, we will have to overcome technical complications which result from the lack of hyperbolicity of the asymptotic steady state.

We use our results to prove the existence of periodic travelling waves accompanying a family of stable solitary waves in the Fermi-Pasta-Ulam lattice, where properties of the latter waves have been recently investigated by Pego and Friesecke [8]. As we will show, these waves persist under small reversible perturbations of the FPU lattice, where they induce a family of solitary waves.
\end{abstract}

\section{Introduction}

We investigate the set of bounded travelling waves near solitary wave solutions of Hamiltonian lattice differential equations. Our work contributes to the theoretical understanding of travelling waves in lattice differential equations and provides an interesting method for proving the existence of periodic travelling waves in the FPU lattice via bifurcation theory. In order to clearly separate our results concerning the theoretical understanding of the dynamics near solitary waves of general lattices from the results which are only valid within the framework of the Fermi-Pasta Ulam lattice, we divide the introduction into two sections. Let us start with the theoretical part.

\subsection{The detection of travelling waves near solitary waves in LDEs}

We want to investigate one-dimensional lattice differential equation (LDE)

$$
\partial_{t} u^{i}(t)=F\left(u^{i-M}(t), \ldots, u^{i}(t), \ldots, u^{i+M}(t)\right),
$$


where each oscillator interacts with its $M$ nearest neighbors. For the moment, let us not assume any additional structure in (1). Interesting solutions are travelling waves. These are solutions of the form $u^{i}(t)=\psi(i-c t), c \neq 0$, which solve a non-trivial advance delay equation

$$
-c \psi^{\prime}(\tau)=F(\psi(\tau-M), \ldots, \psi(\tau), \ldots, \psi(\tau+M)),
$$

where $\tau=i-c t$. In order to solve the associated initial value problem, we have to specify a function on the interval $[-M, M]$ and typically one considers initial data in the space $C^{0}:=C^{0}\left([-M, M], \mathbb{R}^{N}\right)$. However, the initial value problem is ill-posed [18]!

Advance delay equations have been studied almost exclusively over the past ten years $[13,14,15,18,27,29,30]$ among with the pioneering work [32]. Let us now assume the existence of a solitary wave solution

$$
u^{i}(t)=\psi\left(i-c_{*} t\right)
$$

where the profile $\psi(\tau)$ induces a homoclinic solution of (2). We recall that such solutions are globally defined and approach a specific steady state, say zero, as $\tau \rightarrow \pm \infty$. Although the travelling wave equation (2) is ill-posed, existence proofs of solitary waves for broad classes of lattice differential equations relying on variational methods $[7,1]$, center manifold reductions $[20,21]$ or perturbation methods [8] are known.

It is now the aim of this work to provide a method of detecting bounded solutions of $(2)$ for $c \approx c_{*}$ close to the homoclinic orbit. Via ansatz (3) such solutions induce travelling waves with wave speed $c$ of the original lattice differential equation. Depending on the shape of the profile these waves can then be periodic travelling waves (the profile is periodic) or multipulse solutions (the profile possesses finitely many humps) for example.

In order to present our results, let us recall the main result of [17] on which we will build upon. For simplicity, the reader should think of the travelling wave equation as an ordinary differential equation for the moment. It has then been shown in [17] that generically the existence of $2 \omega$ periodic solutions near $\psi$ is equivalent to a zero of $\xi=\xi(\omega, c)$ for some specific function

$$
\xi: \mathbb{R} \times\left(c_{*}-\varepsilon, c_{*}+\varepsilon\right) \rightarrow \mathbb{R} .
$$

Furthermore, it could be constructed a bifurcation function $\tilde{\xi}$ such that the existence of multipulse solutions near the solitary wave is equivalent to a zero of $\tilde{\xi}: \mathbb{R}^{k-1} \times\left(c_{*}-\varepsilon, c_{*}+\varepsilon\right) \rightarrow \mathbb{R}^{k}$. Here, multipulse solutions refer to travelling waves of (1) that induce homoclinic solutions in the travelling wave equation which intersect a local Poincaré section $k$ times for some $k \geq 2$. In either case, the equations $\xi=0$ and $\tilde{\xi}=0$ should be seen as bifurcation equations, which, depending on the number of independent variables and equations, determine the "dimension" of the bifurcation problem. Likewise, we can use these equations in order to investigate the persistence of the homoclinic solution $\psi$ whenever an external parameter is varied. This has be done in the context of reversible LDEs in [16]. Roughly speaking, the bifurcation functions in [17] were obtained by constructing suitable solutions of (2), which are defined and 
close to the primary homoclinic solution $\psi$ on suitable large time intervals $\left[-\omega_{j}, \omega_{j}\right]$. Moreover, these $j$-dependent solutions have been constructed in such a way that a concatenation of them defines a globally piecewise smooth solution of (2) undergoing discontinuities only along a finite-dimensional vector space, which determines the number of scalar bifurcation equations.

In the context of ordinary differential equations, the described reduction procedure is known as Lin's method [2, 23, 22, 24, 25, 26, 33] and was extended only recently to the context of advance delay differential equations [17] in full generality. As a technical issue, hyperbolicity of the asymptotic steady state zero was assumed in the latter work [17]. Here, hyperbolicity refers to the absence of purely imaginary eigenvalues of the linearization at the asymptotic steady state zero (where the reader should again think of (2) as an ordinary differential equation). However, such an assumption is often violated in the framework of Hamiltonian LDEs like the Fermi-Pasta-Ulam lattice and the Klein Gordon lattice [16, 20, 21]. Moreover, most existence results of solitary waves such as the result of Friesecke and Wattis [7] rely on variational methods and refer to equations where the hyperbolicity assumption is violated. It is therefore desirable to extent Lin's method also to the context of lattice differential equations, where hyperbolicity of the steady state may be absent as in the Fermi-Pasta-Ulam lattice. That this is possible, is the main result of this work. Our main result provides the existence of solutions of the travelling wave equation, which stay near the homoclinic solution $\psi$ undergoing possible jumps along a fixed $(1+m)$-dimensional vector space. Hence, if these jumps vanish for some sequence $\left\{\omega_{i}\right\}_{i}$ then we obtain a globally defined solution of (2). The main difference of theorem 1 compared to the results in [17] stems from the $m$-dimensional center eigenspace of the linearization at the steady state. This difference manifests itself in the fact that the number of bifurcation equations increases to $m+1$ (compare with property b) of the theorem below). Indeed, in the hyperbolic scenario $m=0$ the jump functions $\xi_{j}$ generically take values in a one-dimensional vector space which reflects the fact the the homoclinic solution itself is a codimension-one-phenomenon (i.e. the sum of tangent spaces of stable and unstable manifold has codimension one in the ambient space). In the nonhyperbolic case there are now $m$ additional variables $\eta_{1}^{j}, \ldots, \eta_{m}^{j}$ appearing in the bifurcation functions $\xi_{j}$. We can think of these $m$ variables as directions within a Poincaré section, for which we could not a priori solve our bifurcation equations.

We now want to state the main result of this article and we refer to theorem 3 for a formulation which lists all assumptions explicitly. For the statement of this result, we represent $D F(0)$ for any $\phi \in C^{0}$ in the form

$$
D F(0) \phi=\sum_{j=-M}^{M} L^{j} \phi(j)
$$

for suitable $M \times M$-matrices $L^{j}$.

\section{Theorem 1}

Assume that there exists a homoclinic solution of (2) for $c=c_{*}$ to the steady 
state zero such that the characteristic function

$$
\operatorname{det}\left(\lambda \cdot i d-\frac{1}{c_{*}} \sum_{j=-M}^{M} L^{j} e^{j \lambda}\right)
$$

possesses $m$ purely imaginary zeros $\lambda$ counting multiplicity (in the case of ODEs this means that the linearization at zero possesses a $m$-dimensional center eigenspace). Then, for every $\delta_{0}$ under generic assumptions there exists positive constants $\omega_{*}, \delta_{*}$ and parameters $\eta_{1}^{j}, \ldots, \eta_{m}^{j} \approx 0$ with the following properties. For all $c \in B_{\delta_{*}}\left(c_{*}\right)$ and $\left\{\omega_{j}\right\}_{j \in \mathbb{Z}}$, with $\omega_{j} \in\left(\omega_{*}, \infty\right) \cup\{\infty\}$ for all $j \in \mathbb{Z}$, there are ( $\eta_{k}^{j}$-dependent) functions

$$
\begin{array}{ll}
x_{+}^{j}: & {\left[-M, M+\omega_{j}\right) \rightarrow \mathbb{R}^{N},} \\
x_{-}^{j} & :\left[-M-\omega_{j-1}, M\right) \rightarrow \mathbb{R}^{N}, \quad j \in \mathbb{Z},
\end{array}
$$

such that

a) $x_{+}^{j}, x_{-}^{j}$ solve $(6)$ on $\left(0, \omega_{j}\right)$ and $\left(-\omega_{j-1}, 0\right)$, respectively (see also section 3 for a precise definition of a solution).

b) $\left.x_{+}^{j}\left(\omega_{j}+\bullet\right)\right|_{[-M, M]}=\left.x_{-}^{j+1}\left(-\omega_{j}+\bullet\right)\right|_{[-M, M]}$ in $C^{0}\left([-M, M], \mathbb{R}^{N}\right)$.

c) The value

$$
\xi_{j}\left(\left\{\omega_{j}\right\}_{j}, c, \eta_{1}^{j}, \ldots, \eta_{m}^{j}\right):=\left.x_{+}^{j}(\cdot)\right|_{[-M, M]}-\left.x_{-}^{j}(\cdot)\right|_{[-M, M]} \in C^{0}\left([-M, M], \mathbb{R}^{N}\right)
$$

lies in a $j$-independent $(m+1)$-dimensional vector space. For the smoothness properties of $\xi_{j}$ we refer to theorem 3 .

d) For any given bounded interval $\mathcal{I}$, the values $x_{+}^{j}(\tau), x_{-}^{j+1}(\tau)$ approaches the value $\psi(\tau)$ uniformly with respect to $\tau \in \mathcal{I}$ as $\omega_{j} \rightarrow \infty$.

e) It is true that

$$
\sup _{\left[-M, M+\omega_{j}\right]}\left|x_{+}^{j}(\tau)-\psi(\tau)\right|<\delta_{0}, \quad \sup _{\left[-M-\omega_{j-1}, M\right]}\left|x_{-}^{j}(\tau)-\psi(\tau)\right|<\delta_{0} .
$$

Finally, the solutions $\left\{x_{ \pm}^{j}\right\}_{j \in \mathbb{Z}}$ are unique once the parameters $\left\{\eta_{k}^{j}\right\}_{j \in \mathbb{Z}}$ are fixed.

In the course of constructing the jump functions we will derive an explicit representation for the $\xi_{j}$, see for example lemma 12. This expression mainly involves integral equations similar to the variation-of-constants formula and can be very helpful when calculating a partial derivative of the jump functions; see for example [17] where the derivative with respect to the wave speed $c$ has been investigated. The formula of the jump functions also indicates that in particular geometric informations are important to investigate the zero set of the $\xi_{j}$ 's with respect to the flight times $\omega_{j-1}, \omega_{j}$. With "geometric" information we mean informations regarding the relation of center stable and center unstable manifolds along the homoclinic solution $\psi$, i.g. how these manifolds 
are twisted along the homoclinic solution $\psi$ or how the center part of these manifolds approaches the steady state and we refer to [24, 25, 26, 22] for more information and background in this respect in the framework of ordinary differential equations. However, there is yet another nice property of the jump functions which shows up when we consider time-reversible or Hamiltonian LDEs: Here, more structure is present in the bifurcation equations $\xi_{j}=0$ that eases the analysis of the zero-set. For example, if the LDE is reversible and the homoclinic solution intersects the fixpoint space of the reversibility $R$, then in the study of periodic solutions the associated jump function will only take values in the fixpoint space of $-R$, see lemma 12 . This property is important since it helps significantly to reduce the number of bifurcation equations a priori without computation and we refer to section 6 in order to see how this is done in the context of the Fermi-Pasta-Ulam lattice. A straightforward application of this last observation is the blue sky catastrophe; i.e. the coexistence of a one-parameter family of periodic orbits close the homoclinic orbit in the hyperbolic case $m=0$, see $[3,5,17]$, where the reversible structure forces all jump functions to vanish identically. Also conserved quantities, i.e. functions which stay constant along solutions, have implications for the investigation of periodic functions. Typically, each conserved quantity then reduces the number of bifurcation functions by one, see the argumentation after hypothesis 5 . We use these observations to prove the existence of periodic travelling waves in the Fermi-Pasta-Ulam lattice as explained in more detail in the next section.

\subsection{Periodic travelling waves in the Fermi-Pasta-Ulam lattice}

An important lattice differential equation is the Fermi-Pasta Ulam equation

$$
\ddot{u}_{n}(t)=\mathcal{V}\left(u_{n+1}(t)-u_{n}(t)\right)-\mathcal{V}\left(u_{n}(t)-u_{n-1}(t)\right),
$$

where $u_{n}(t) \in \mathbb{R}$ and the potential $\mathcal{V} \in C^{3}$ satisfies $\mathcal{V}(0)=\mathcal{V}^{\prime}(0)=0$ $\mathcal{V}^{\prime \prime}(0) \neq 0$. Its popularity stems from the fifties, where Fermi, Pasta and Ulam used this model to show the relaxation to equipartition of the distribution of energy among modes [6]. Surprisingly, their numerical simulation yielded an unexpected result: At least at low energy, the energy of the system remained confined among the initial modes instead of spreading to all modes. Concerning exact dynamics in the Fermi-Pasta-Ulam lattice it was proved only recently by Friesecke and Wattis [7] that (4) exhibits solitary wave solutions, i.e. localized coherent modes. Here, localization refers to the fact that the relative displacements of the adjacent lattice sites $r_{n}(t):=u_{n+1}(t)-u_{n}(t)$ decay to zero for fixed $n$ as $t \rightarrow \pm \infty$. Note that $r_{n}(t)$ solves the equation

$$
\ddot{r}_{n}(t)=\mathcal{V}\left(r_{n+1}(t)\right)-2 \mathcal{V}\left(r_{n}(t)\right)+\mathcal{V}\left(r_{n-1}(t)\right) .
$$

However, no informations about stability or long-time behaviour of nearby initial data, was obtained. But very recently Friesecke and Pego $[8,9,10,11]$ have constructed a family of supersonic solitary waves $r_{n}$ of (5) which appear stably in the lattice equation. More precisely, it was shown that each initial 
value close to $r_{n}$ in a proper sense induces a solution of (5) that asymptotically approaches a translated copy of the original solitary wave (typically with slightly different wave speed).

Since the waves $r_{n}$ have the form $r_{n}(t)=\psi(n-c t)$ for some $c \neq 0$ the associated profile $\psi(\tau)$ solves the travelling wave equation which is an advance delay and in fact induces a homoclinic solutions. Equation (5) is time-reversible; hence the travelling wave equation is also (we refer to section 3.3 for a definition of reversibility in this context). We are now interested in the question whether the homoclinic solution, which is induced by the solitary wave, is accompanied by periodic solutions. Note that these induce periodic travelling wave solutions of the original lattice equation (5) via the travelling wave ansatz (3). Thinking of a reversible ordinary differential equation, such a scenario would typically occur provided the steady state approached by the homoclinic solution is hyperbolic $[3,5,17]$. However, for the travelling wave equation of the FPU-lattice two major differences arise: First of all the travelling wave equation is an advance delay equation and in particular ill-posed. Moreover the linearization at the steady state zero possesses a two-dimensional center eigenspace, see section 6.1.1, and therefore the steady state is not hyperbolic. However, as we show in section 6 the reversible and Hamiltonian structure are strong enough to enforce the existence of a family of periodic solutions of large period that are close to the primary homoclinic solution, see theorem 4 . The term "close" here refers to orbitally close meaning that the orbits of the periodic solutions are close to the homoclinic orbit. This does not contradict the asymptotic stability of the solitary wave as a solution of (38), where stability is defined in terms of suitably weighted norms. In particular, the distance of two solutions can be large with respect to some fixed weighted norm but their orbits can be close to each other.

In the course of proving our main result, we first investigate the existence of periodic travelling waves in equation (5) near any solitary wave satisfying generic assumptions. In particular, we do not assume that the primary solitary wave has to be small or close to an equilibrium in some sense. In this respect, our bifurcation scenario is rather general and in principal not restricted to the Fermi-Pasta-Ulam lattice. We will then validate all necessary assumptions for a special family of solitary waves in section 6.2 , whose properties have recently been investigated by Friesecke and Pego [8, 9, 10, 11]. Moreover, we show that the periodic waves persist under small reversible perturbations of the FPU lattice and induce a family of solitary waves in the perturbed FPU lattice. For more details of this bifurcation scenario and more properties of the bifurcating waves we refer to section 6 .

The existence of periodic travelling waves of the Fermi-Pasta-Ulam lattice (5) has also been shown by Pankov [31] using variational methods. Let us also mention that Iooss and Kirchgässner [21] showed the existence of oscillatory solutions in (4), which do not necessarily induce periodic travelling waves of (5). In any case, our existence proof is important in view of various aspects. First of all, our results cover a slightly more general class of potentials $\mathcal{V}$ than considered in [31], see theorem 4. Furthermore, our constructed family of periodic waves (of fixed period) depends differentiable on the wave speed - a 
property which cannot be deduced easily from variational methods. But even more important, the bifurcating periodic waves are related to the family of stable solitary waves in the sense that the associated travelling wave profiles are orbitally close. In this respect we also expect that our methods are able to relate stability properties of the bifurcating periodic travelling wave to the stability of the original wave; first steps toward this directions have already been achieved by Pego and Friesecke [8] and Benzoni-Gavage et al [12]. With the methods introduced in this work, we aim at extending these results in the near future and thereby introducing a stability analysis which resembles the theory from Sandstede and Scheel in the context of reaction-diffusion equations $[34,35,36]$. Finally, we want to emphasize that our approach shows how dynamical systems methods and in particular bifurcation theory can be applied in order to detect periodic waves near solitary waves; a method which to the knowledge of the author has not been used within the framework of Hamiltonian lattice differential equations before.

\subsection{Organisation of the article}

The set up of the article is as follows. We will introduce some notation in the next section. In section 4 we will then set up our problem in a suitable functional analytic framework, where we collect some background concerning linear and nonlinear advance-delay equations as well as the abstract formulation in section 3. The main result is stated in chapter 5. In section 5.1.1 we give a brief outline of the proof of this result. Its rigorous proof is then postponed to the next section 5.1. We apply our reduction procedure to prove the existence of periodic travelling waves in the Fermi-Pasta-Ulam lattice in chapter 6 . In section 6.3 we also will discuss the existence of linearly stable solitary waves in slightly perturbed FPU-lattices which still are reversible.

\section{Important function spaces}

We define the function $x_{t} \in L^{2}\left([-M, M], \mathbb{R}^{N}\right)$ for an integrable function $x$ : $\mathbb{R} \rightarrow \mathbb{R}^{N}$. This function is given by $x_{t}(\theta):=x(t+\theta)$ for any $t \in \mathbb{R}$. Hence, for the rest of this work $x_{t}$ will not mean a derivative!

We also abbreviate the space $C^{0}\left([-M, M], \mathbb{R}^{N}\right)$ often by $C^{0}$. Also the following spaces will be frequently used throughout this paper:

$$
\begin{aligned}
Y & :=\mathbb{R}^{N} \times L^{2}\left([-M, M], \mathbb{R}^{N}\right), \\
X & :=\left\{(\xi, \varphi) \in Y \mid \varphi \in H^{1}\left([-M, M], \mathbb{R}^{N}\right) \text { and } \varphi(0)=\xi\right\} \\
\tilde{X} & :=\left\{(\xi, \phi) \in \mathbb{R}^{N} \times C^{0}\left([-M, M], \mathbb{R}^{N}\right): \phi(0)=\xi\right\}
\end{aligned}
$$

As a convention, if subspaces are furnished with an additional , they are regarded as subspaces of $\tilde{X}$ with the induced norm. 


\section{Background on advance delay equations}

In this section we collect some results concerning the abstract formulation of advance-delay equations which will be used in the sequel.

\subsection{The abstract formulation of advance delay equa- tions}

Let us consider the traveling wave equation

$$
\dot{x}(t)=\frac{1}{c} F(x(t-M), \ldots, x(t), \ldots, x(t+M))
$$

for $c \neq 0$ where we assume from now on that $F \in B C^{2}\left(\mathbb{R}^{N(2 M+1)} \times \mathbb{R} ; \mathbb{R}^{N}\right)$ and $F(0)=0$. We make the following definition.

\section{Definition 1 (Solution)}

We call a function $x \in L^{2}\left([-M, \tau), \mathbb{C}^{N}\right)$ a solution of (6) for some $M<\tau \leqslant \infty$ to the initial condition $\phi \in L^{2}\left([-M, M], \mathbb{C}^{N}\right)$, if $x \in H_{l o c}^{1}([0, \tau), \mathbb{C}), x_{0}=\phi$ and $(6)$ is satisfied for almost every $t \in[0, \tau)$.

However, instead of working with (6) directly, we prefer to work with the abstract equation

$$
\begin{aligned}
\dot{U}(t) & =\mathcal{F}(\xi(t), \phi(t, \cdot)) \\
& =\left(\begin{array}{c}
\left.\frac{1}{c} F(\phi(t,-M), \ldots, \phi(t, 0), \ldots, \phi(t, M))\right) \\
\partial_{\theta} \phi(t, \theta)
\end{array}\right) .
\end{aligned}
$$

This approach has first been used in [20, 21] although with a slightly different choice of state spaces $X, Y$. We refer the reader to section three of [17] for some motivations of studying the abstract equation or even to the nice text book [4], which addresses this topic for delay differential equations.

\section{Definition 2}

- We call a continuous function $U(t):\left[t_{1}, t_{2}\right) \rightarrow Y$ a solution of (7) on $\left(t_{1}, t_{2}\right)$, where $-\infty<t_{1}<t_{2} \leqslant \infty$, if $t \rightarrow U(t)$ is continuous regarded as a map on $\left(t_{1}, t_{2}\right)$ with values in $X$, if $t \rightarrow U(t)$ is differentiable regarded as a map on $\left(t_{1}, t_{2}\right)$ with values in $Y$ and $(7)$ is satisfied on $\left(t_{1}, t_{2}\right)$.

- We call a differentiable function $U(t):\left(-\infty, t_{2}\right) \rightarrow Y$ a solution of $(7)$ on $\left(-\infty, t_{2}\right)$ and $t_{2} \in \mathbb{R}$, if $t \rightarrow U(t)$ is continuous regarded as a map on $\left(-\infty, t_{2}\right)$ with values in $X$ and $(7)$ is satisfied on $\left(-\infty, t_{2}\right)$.

- We call a continuous function $U:\left[t_{1}, t_{2}\right) \rightarrow \tilde{X}$ a weak solution of (7), if

$$
U(t)=\left(x(t), x_{t}\right)
$$

for some function $x \in C^{0}\left(\left[t_{1}-M, t_{2}+M\right), \mathbb{R}^{N}\right) \cap C^{1}\left(\left(t_{1}, t_{2}+M\right), \mathbb{R}^{N}\right)$ which solves the equation $\dot{x}(t)=F\left(x_{t}, \lambda\right)$ on $\left(t_{1}, t_{2}\right)$. 
The next lemma clarifies the connection between solutions of (7) and our original equation (6). The proof can be found in $[13,14]$.

\section{Lemma 1}

Let

$$
U(t)=\left(\begin{array}{c}
\xi(t) \\
\varphi(t)(\cdot)
\end{array}\right)
$$

be a solution of (7) on $\left(t_{1}-M, t_{2}+M\right)$. Then $\varphi(t)(\theta)=\xi(t+\theta)$ for all $t \in\left(t_{1}-M, M+t_{2}\right)$ and $\theta \in[-M, M]$ with $t+\theta \in\left(t_{1}-M, t_{2}+M\right)$. Furthermore $\xi(t)$ solves (6) on the interval $\left(t_{1}, t_{2}\right)$.

\subsection{Linear equations}

In this section we collect some known facts about linear functional differential equations of mixed type which we will use in the sequel, see also [29, 18]. We investigate the linear equation

$$
\dot{y}(t)=L(t) y_{t},
$$

where we have set $y_{t}(\theta):=y(t+\theta)$ for any $\theta \in[-M, M]$ and where

$$
L(t) \phi=\sum_{j=-M}^{M} L_{j}(t) \phi(j)
$$

for $\phi \in C^{0}\left([-M, M], \mathbb{R}^{N}\right)$ and some $L_{j}(\cdot) \in B C^{0}\left(\mathbb{R}, L\left(\mathbb{C}^{N}, \mathbb{C}^{N}\right)\right)$ where we assume that $\lim _{t \rightarrow \infty} L(t)=L_{+}$and $\lim _{t \rightarrow-\infty} L(t)=L_{-}$exist. As in the nonlinear case we can relate equation (8) to the abstract equation

$$
\partial_{t} V(t)=\mathcal{A}(t) V(t),
$$

where the linear operator $\mathcal{A}(t): X \subset Y \rightarrow Y$ is defined by

$$
\mathcal{A}(t)\left(\begin{array}{c}
\xi \\
\varphi
\end{array}\right)=\left(\begin{array}{c}
L(t) \varphi \\
\partial_{\theta} \varphi
\end{array}\right)
$$

for $(\xi, \varphi) \in X$. Let us set $\mathcal{A}_{+}:=\lim _{t \rightarrow \infty} \mathcal{A}(t)$ (i.e. where $L(t)$ in the definition of $\mathcal{A}(t)$ is replaced by $\left.L^{+}:=\lim _{t \rightarrow \infty} L(t)\right)$. Then it is known that the spectrum of the densely defined operator $\mathcal{A}_{+}: X \subset Y \rightarrow Y$ only consists of eigenvalues of finite multiplicity. Moreover, an element $\lambda_{*} \in \mathbb{C}$ is in $\operatorname{spec}\left(\mathcal{A}_{+}\right)$, if the characteristic function vanishes at $\lambda_{*}$, that is, if

$$
\operatorname{det}(\triangle(\lambda)):=\operatorname{det}\left[\lambda \cdot i d-\sum_{j=-M}^{M} L_{j}^{+}\left(e^{+j \lambda} \cdot i d\right)\right]=0
$$

for $\lambda=\lambda_{*}$, where $L_{j}^{+}:=\lim _{t \rightarrow \infty} L_{j}(t)$. Furthermore, the algebraic multiplicity of $\lambda_{*}$ as an eigenvalue of $\mathcal{A}_{+}$(which is the dimension of its generalized eigenspace) coincides with the order of $\lambda_{*}$ as a zero of $\operatorname{det} \triangle(\cdot)$; we refer to $[13,18]$ for proofs of these statements. 


\section{Definition 3}

We call a linear equation $\dot{x}(t)=L x_{t}$ ( respectively $\dot{V}=\mathcal{A} V$ ) hyperbolic for some $L \in L\left(C^{0}, \mathbb{C}^{N}\right)$ (respectively $\mathcal{A}=\left(L, \partial_{\theta}\right) \in L(X, Y)$ ), if the characteristic equation

$$
\operatorname{det} \triangle(\lambda):=\operatorname{det}\left(\lambda \cdot i d-\left(\sum_{k=1}^{m} L_{k} e^{\lambda k}\right)\right)=0
$$

does not possess purely imaginary zeros $\lambda=i s$ with $s \in \mathbb{R}$.

\subsubsection{Center dichotomies}

In our situation we will encounter the case that the variational equation

$$
\dot{y}(t)=L(t) y_{t}
$$

is asymptotically constant but not asymptotically hyperbolic with $L_{+}=L_{-}$ such that the generalized center eigenspace of $\mathcal{A}_{+}$is $m$-dimensional. Indeed, later on we will consider the specific case

$$
L(t):=\frac{1}{c_{*}} D_{1} F(h(t-M), \ldots, h(t), \ldots, h(t+M)) .
$$

However, we still can prove the existence of solution operators for the associated abstract equation related to an exponentially decreasing and growing part, respectively. This is the content of the next theorem and we refer to $[13,18]$ for a proof.

\section{Theorem 2 (Center stable dichotomies on $\mathbb{R}_{+}$)}

Assume that

$$
\operatorname{det}\left(L_{-M}^{+}(\cdot)\right) \text { and } \operatorname{det}\left(L_{M}^{+}(\cdot)\right)
$$

do not vanish identically on any nontrivial interval of $\mathbb{R}$. Moreover, suppose that the equation $\dot{V}(t)=\mathcal{A}_{+}(t) V(t)$ is nonhyperbolic. Then (10) possesses a center stable dichotomy on $\mathbb{R}_{+}$. That is, there exist constants $K, \alpha>0$ and a family of strongly continuous projections $P^{c s}(t): Y \rightarrow Y, t \geqslant 0$, with the following properties. For any $\delta>0$ it is true that for $U \in Y$ and $t_{0} \geqslant 0$

i) there exists a continuous function $\Phi_{+}^{c s}(\cdot, \cdot) U:\left\{\left(t, t_{0}\right): t \geqslant t_{0} ; t, t_{0} \geqslant 0\right\} \rightarrow$ $Y$, such that $\Phi_{+}^{c s}\left(t_{0}, t_{0}\right) U=P^{c s}\left(t_{0}\right) U$. Moreover, $\Phi^{c s}\left(t, t_{0}\right) U \in R g\left(P^{c s}(t)\right)$ and $\left|\Phi_{+}^{c s}\left(t, t_{0}\right) U\right|_{Y} \leqslant K e^{\delta\left|t-t_{0}\right|}|U|_{Y}$ for all $t \geqslant t_{0} \geqslant 0$.

ii) There exists a continuous function $\Phi_{+}^{u}(\cdot, \cdot) U:\left\{\left(t, t_{0}\right): t \leqslant t_{0} ; t, t_{0} \geqslant\right.$ $0\} \rightarrow Y$, such that $\Phi_{+}^{u}\left(t_{0}, t_{0}\right) U=\left(i d-P^{c s}\left(t_{0}\right)\right) U$. Moreover, $\Phi_{+}^{u}\left(t, t_{0}\right) \in$ $\operatorname{Rg}\left(i d-P^{c s}(t)\right)$ and $\left|\Phi_{+}^{u}\left(t, t_{0}\right) U\right|_{Y} \leqslant K e^{-\alpha\left|t-t_{0}\right|}|U|_{Y}$ for all $t_{0} \geqslant t \geqslant 0$.

In the special case $U \in X$ the functions $t \mapsto \Phi_{+}^{c s}\left(t, t_{0}\right) U$ and $t \mapsto \Phi_{+}^{u}\left(t, t_{0}\right) U$ define classical solutions of (10) on their domain of definition. Moreover, if $U \in R g\left(P^{c s}\left(t_{0}\right)\right)$ with $U=(\zeta, \phi(\cdot))$ the map $\Phi_{+}^{c s}\left(t, t_{0}\right) U$ is of the form $\left(x(t), x_{t}\right)$ for $t>t_{0}, \Phi_{+}^{c s}\left(t_{0}, t_{0}\right) U=U$ and $x(\cdot)$ defines a solution of (8) with $x_{0}=\phi$. An analogous statement holds for $\Phi_{+}^{u}\left(t, t_{0}\right) U$.

However, even more is true (see also lemma 5 in [16]): 


\section{Lemma 2}

Assume that the asymptotic characteristic equation

$$
\operatorname{det}(\triangle(\lambda)):=\operatorname{det}\left[\lambda \cdot i d-\sum_{j=-M}^{M} L_{j}^{+}\left(e^{+j \lambda} \cdot i d\right)\right]
$$

of (8) possesses exactly $m$ purely imaginary zeros $\lambda$ counting multiplicities. Then there exists an $m$-dimensional subspace $E_{+}^{c}(0) \subset E_{+}^{c s}(0)$ and a closed complement $E_{+}^{s}(0) \subset E_{+}^{c s}(0)$, such that for any $U \in E_{+}^{c s}(0)$

$$
\left.\Phi_{+}^{c s}(t, 0)\right|_{E_{+}^{s}(0)} U
$$

decays exponentially as $t \rightarrow \infty$ if and only if $U \in E_{+}^{s}(0)$.

Hence, it is possible to "factor out" the $m$-dimensional center part of the center stable solution operator.

Analogously we can prove the existence of center-unstable dichotomies on $\mathbb{R}^{+}$ as well as the existence of center stable- and center unstable dichotomies on $\mathbb{R}_{-}$.

\subsection{Reversible equations}

We call the abstract equation (7) reversible if

$$
\mathcal{R} \mathcal{F}(U)=-\mathcal{F}(\mathcal{R} U)
$$

for any $U=(\xi, \phi) \in X$, where the linear map $\mathcal{R}: Y \rightarrow Y$ is defined by

$$
\mathcal{R}(\xi, \phi(\theta)):=(R \xi, R[\mathcal{S} \phi(\cdot)])=(R \xi, R \phi(-\theta))
$$

and $(\mathcal{S} \phi)(\theta):=\phi(-\theta)$ for any $\phi \in C^{0}\left([-M, M], \mathbb{R}^{N}\right)$, we call the abstract equation (7) reversible. Here, we assume that $R \in L\left(\mathbb{R}^{N}\right)$ can be represented in the form

$$
R=P_{i_{1}} \circ P_{i_{2}} \circ \ldots \circ P_{i_{n}},
$$

where the reflection $P_{i}, 1 \leqslant i \leqslant N$, is defined by

$$
P_{i}\left(x^{1}, \ldots, x^{N}\right) \mapsto\left(x^{1}, \ldots, x^{i-1},-x^{i}, x^{i+1}, \ldots, x^{N}\right) .
$$

An important space is the fixpoint space $\operatorname{Fix}(\mathcal{R})$, where

$$
\operatorname{Fix}(\mathcal{R}):=\{U \in Y: \mathcal{R} U=U\} .
$$

\subsection{Poincaré sections}

Finally, we will state a result which allows us to construct suitable $\mathcal{R}$-invariant Poincaré-sections near $H(\tau)$, if $H$ denotes a symmetric homoclinic solution. Here, symmetric means that $H\left(\tau_{*}\right) \in \operatorname{Fix}(\mathcal{R})$ for some $\tau_{*} \in \mathbb{R}$. In order to state the next result, let us consider the linearization (10) along a homoclinic solution. If the asymptotic steady state is non-hyperbolic there exist dichotomies 
with solution operators $\Phi_{+}^{c s}(t, s), t \geq s \geq 0$ and $\Phi_{-}^{u}(t, s), t \leq s \leq 0$. We have shown in [15] that in this case

$$
\operatorname{Rg}\left(\Phi_{+}^{c s}(0,0)\right)+\operatorname{Rg}\left(\Phi_{-}^{u}(0,0)\right)
$$

is closed and possesses a finite-dimensional complement in $Y$, which typically has dimension one. More precisely, the codimension of this space coincides with the number of (up to scalar multiples) globally defined solutions of (8), which decay exponentially as $t \rightarrow-\infty$ and grow at most with small exponential rate as $t \rightarrow \infty$ (i.e. the grow rate is less than $e^{\delta|t|}$ as $t \rightarrow \infty$ for any fixed $\delta>0$ ). Similarly, also $\operatorname{Rg}\left(\left.\Phi_{+}^{c s}(0,0)\right|_{\tilde{X}}\right)+\operatorname{Rg}\left(\left.\Phi_{-}^{u}(0,0)\right|_{\tilde{X}}\right)$ is closed and then possesses a complement in $\tilde{X}$ with the same dimension, see [14]. Let us assume now there exists an $m$-dimensional complement of $E_{+}^{s}(0)=\operatorname{Rg}\left(\Phi_{+}^{s}(0,0)\right)$ in the space $E_{+}^{c s}(0) \subset Y$. We can then state the next result whose proof can be found in [15].

\section{Lemma 3 (Poincaré sections)}

There exist subspaces $\tilde{\mathcal{E}}_{+}^{s}(0) \subset \operatorname{Rg}\left(\Phi_{+}^{s}(0,0)\right)$ and $\tilde{\mathcal{E}}_{-}^{u}(0) \subset R g\left(\Phi_{-}^{u}(0,0)\right)$ which are complementary to $\operatorname{span}\langle\mathcal{F}(H(0))\rangle$ and that are closed with respect to the $\tilde{X}$-norm. Moreover, for any complement $\tilde{K}$ of the sum $\tilde{\mathcal{E}}_{+}^{s}(0)+\tilde{\mathcal{E}}_{-}^{u}(0)+$ $\operatorname{span}\langle\mathcal{F}(H(0))\rangle$ (in particular, $\tilde{K}$ is finite dimensional), the space

$$
\tilde{\Gamma}:=\tilde{K} \oplus \tilde{\mathcal{E}}_{+}^{s}(0) \oplus \tilde{\mathcal{E}}_{-}^{u}(0)
$$

is closed with respect to the $\tilde{X}$-norm and defines a Poincaré-section at $H(\tau)$ via $\tilde{\Sigma}:=H(\tau)+\tilde{\Gamma}$. If $\tilde{K}$ is chosen to be $\mathcal{R}$-invariant, then also the space $\tilde{\Gamma}$ is $\mathcal{R}$-invariant.

\section{Set up}

Instead of working with the original travelling wave equation

$$
c u^{\prime}(t)=F(u(t-M), \ldots, u(t), \ldots, u(t+M))
$$

which we will also write in the form $c u^{\prime}(t)=F\left(u_{t}\right)$, we will from now on consider the abstract equation

$$
\begin{aligned}
\dot{U}(t) & =\mathcal{F}((y(t), \phi(t, \cdot)), c) \\
& =\left(\begin{array}{c}
\left.\frac{1}{c} F(\phi(t,-M), \ldots, \phi(t, 0), \ldots, \phi(t, M))\right) \\
\partial_{\theta} \phi(t, \theta)
\end{array}\right),
\end{aligned}
$$

in the space $Y$, where $(y(t), \phi(t, \cdot)) \in X$ for each $t$. Concerning solutions of (17), let us emphasize that in particular weak solutions $U(t)=\left(y(t), y_{t}\right)$ of $(17)$ (as defined in section 3.1) play an important role, since they arise naturally as a consequence of our functional analytic set up; especially when working with integral equations like variants of the variation-of-constants formula [17]. As defined in section 3.1, weak solutions are functions for which $y:(-M, \infty) \rightarrow$ $\mathbb{R}^{N}$ is continuous and solves the original travelling wave equation $(16)$ on $(0, \infty)$ 
(we may in fact also consider intervals different from $(-M, \infty)$ ). Hence, already weak solutions $U(t)$ on $(0, \infty)$ of the abstract equation then induce by definition classical solutions $\xi$ of the travelling wave equation $(16)$ on $(0, \infty)$. These, however, do not immediately translate back to travelling wave solutions $u^{i}(t)$ of the lattice equation. In fact, we need a globally defined solution $y$ of (16), which then defines a solution $u^{i}$ of (1) via

$$
u^{i}(t)=y(i-c t) .
$$

As a starting point we now make the next assumption, which guarantees the existence of a solitary wave solution of the original travelling wave equation (1).

\section{Hypothesis 1}

Equation (17) possesses a homoclinic solution $H(t)=\left(\psi(t), \psi_{t}\right)$ to the nonhyperbolic steady state zero for $c=c_{*}$ and decays exponentially, i.e.

$$
|\psi(t)|<C e^{-2 \alpha_{*}|t|}
$$

for $|t|$ large enough and some $\alpha_{*}>0$. Moreover, the characteristic equation with respect to $c_{*} v^{\prime}(t)=D F(0) v_{t}$ possesses exactly $m$ purely imaginary zeros counting multiplicity.

Let us stress the fact that the exponential decay-condition is not automatically satisfied, since the homoclinic solution may approach the steady state asymptotically along a center direction for one direction $t \rightarrow \pm \infty$ and hence with algebraic rate. However, in the Fermi-Pasta-Ulam lattice, where we always encounter a line of equilibria, hypothesis 1 is satisfied for supersonic solitary waves where $m=2$ (see section 6 for details). A similar scenario arises, if the linearization at the steady state possesses exactly two simple purely imaginary eigenvalues $\pm i \omega$, which typically lead to the existence of a one-parameter family of small periodic solutions in reversible equations (16) (we refer to [16] for such a result). Hence, any homoclinic solution to this steady state necessarily lies in the strong stable and strong unstable manifold, respectively, and hypothesis 1 is satisfied.

Since we now want to study solutions near the homoclinic solution $H$, it is natural to linearize along the homoclinic solutions $H$. This leads to the equation

$$
\partial_{t} V(t)=\mathcal{A}(t) V(t):=\left(\begin{array}{c}
\frac{1}{c_{*}} D_{1} F\left(\psi_{t}\right) \phi(t, \cdot) \\
\partial_{\theta} \phi(t, \theta)
\end{array}\right),
$$

where $V(t)=(\eta(t), \phi(t, \cdot))$. Let us assume that the assumption (13) is satisfied. Hence, on account of theorem 2 of section 3.2, the equation (18) admits center stable and center unstable dichotomies with associated solution operators $\Phi_{+}^{c s}, \Phi_{+}^{u} \in L(Y, Y)$ and $\Phi_{+}^{c u}, \Phi_{+}^{s} \in L(Y, Y)$ on $\mathbb{R}_{+}$and $\Phi_{-}^{c s}, \Phi_{-}^{u} \in L(Y, Y)$ and $\Phi_{-}^{c u}, \Phi_{-}^{s} \in L(Y, Y)$ on $\mathbb{R}_{-}$.

\section{Notation}

From now on we will often denote by $P_{+}^{c s}(t)=\Phi_{+}^{c s}(t, t), t \geq 0$, and $P_{+}^{s}(t)=$ $\Phi_{+}^{s}(t, t), t \geq 0$, the projections onto the center stable and strong stable space 
associated to a center stable and center unstable dichotomy on $\mathbb{R}_{+}$, respectively. Similarly, the projections $P_{-}^{u}(t), t \leq 0, P_{-}^{c u}(t), t \leq 0$, are defined.

We also denote by $E_{+}^{c s}(t):=\operatorname{Rg} P_{+}^{c s}(t), t \geq 0$ the associated range of the center stable projection, which is by definition a closed subspace of $Y$. Analogously, the spaces $E_{+}^{s}(t), E_{+}^{c u}(t), E_{+}^{u}(t), t \geq 0$, and $E_{-}^{u}(t), E_{-}^{c u}(t), E_{-}^{u}(t), E_{-}^{c s}(t), t \leq 0$ are defined. Note that these subspaces are closed subspaces of $Y$ whereas an additional refers to the spaces as subspaces of $\tilde{X}$ (i.e. for example $\tilde{E}_{+}^{c s}(0)=\operatorname{Rg}\left(\left.P_{+}^{c s}(0)\right|_{\tilde{X}}\right)$ etc. $)$. That these spaces are actually closed in $\tilde{X}$ is proved in [14].

By the results of [13] we also have that the solution operators satisfy

$$
\left.\Phi_{+}^{c s}(t, s)\right|_{\tilde{X}},\left.\Phi_{+}^{u}(s, t)\right|_{\tilde{X}} \in L(\tilde{X}, \tilde{X}), \quad t \geq s \geq 0
$$

and the norms can be bounded as in $i$ ), ii) of theorem 2 with respect to the $\tilde{X}$-norm. Let us make the following generic assumption.

\section{Hypothesis 2}

$H$ is non degenerate, i.e. $E_{+}^{c s}(0) \cap E_{-}^{u}(0)=\operatorname{span}\left\langle\mathcal{F}\left(H(0), c_{*}\right)\right\rangle$.

As a consequence of hypothesis 2, center stable and unstable manifold of zero intersect only along $H$ and the space $\tilde{E}_{+}^{c s}(0)+\tilde{E}_{-}^{u}(0)$ has codimension one in $\tilde{X}$, see lemma 6.1 and remark 6.1 in [16]. Moreover, on account of lemma 2 the space $E_{+}^{s}(0)$ possesses codimension $m$-dimensional in the space $E_{+}^{c s}(0)$ and the same is true with respect to the spaces $\tilde{E}_{+}^{s}(0)$ and $\tilde{E}_{+}^{c s}(0)$.

\section{$5 \quad$ Lin's method in the case of non-hyperbolic steady states}

In this section we want to prove our main result. For the statement of the theorem we recall that $\tilde{\mathcal{E}}_{+}^{s}(0) \subset \tilde{E}_{+}^{s}(0)$ and $\tilde{\mathcal{E}}_{-}^{u}(0) \subset \tilde{E}_{-}^{u}(0)$ denote closed complements of $\operatorname{span}\left\langle\mathcal{F}\left(H(0), c_{*}\right)\right\rangle$ with respect to $\tilde{X}$ and have been defined in lemma 3. On account of hypothesis 1 the generalized center eigenspace with respect to the linearization of (17) at 0 is $m$-dimensional. Taking into account hypothesis 2 we can therefore find an $(1+m)$-dimensional complement $\tilde{K}$ of the closed space $E_{+}^{s}(0)+E_{-}^{u}(0)$ in $Y$, such that

$$
E_{+}^{s}(0)+E_{-}^{u}(0)+\tilde{K}=Y
$$

see lemma 5 in [16]. By the same result we are also allowed to choose $\tilde{K}$ to lie in $\tilde{X}$ which implies that $\tilde{E}_{+}^{s}(0)+\tilde{E}_{-}^{u}(0)+\tilde{K}=\tilde{X}$. We can now formulate our main result.

\section{Theorem 3}

Assume that (13), which assures the existence of center dichotomies for the linearized equation along the homoclinic solution, and the hypotheses 1 and 
2 are satisfied. Fix a one-dimensional complement $\tilde{Z} \subset \tilde{X}$ of $\operatorname{Rg}\left(\left.P_{+}^{c s}(0)\right|_{\tilde{X}}\right)+$ $\operatorname{Rg}\left(\left.P_{-}^{u}(0)\right|_{\tilde{X}}\right)$. Moreover, choose a complement $\tilde{K}^{c} \subset \tilde{X}$ of

$$
\operatorname{Rg}\left(\left.P_{+}^{s}(0)\right|_{\tilde{X}}\right)+\operatorname{Rg}\left(\left.P_{-}^{u}(0)\right|_{\tilde{X}}\right)+\tilde{Z}
$$

and set $\tilde{K}=\tilde{Z}+\tilde{K}^{c}$.

Select a constant $M_{*}>0$ and integers $m_{-}^{j}, m_{+}^{j} \geq 0$ with the property $m_{+}^{j}+$ $m_{-}^{j}=m$ for all $j \in \mathbb{Z}$, where $m=\operatorname{dim}\left(\tilde{K}^{c}\right)$. Then there exist positive constants $\omega_{*}, \delta_{*}, \gamma_{*}$ such that the following holds. For all $c \in B_{\delta_{*}}\left(c_{*}\right)$ and all

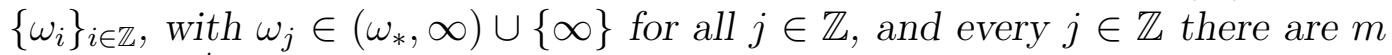
variables $\eta_{1}^{j}, \ldots, \eta_{m}^{j}$ with

$$
\begin{aligned}
\left|\eta_{k}^{j}\right| & \leq M_{*} e^{-\gamma \omega_{j}}, & & k=1, \ldots, m_{+} \\
\left|\eta_{k}^{j}\right| & \leq M_{*} e^{-\gamma \omega_{j-1},} & & k=m_{+}+1, \ldots, m \\
\eta_{k}^{j} & =0, & & \text { if } \omega_{j}=\infty \text { and } k=1, \ldots, m_{+} \\
\eta_{k}^{j} & =0, & & \text { if } \omega_{j-1}=\infty \text { and } k=m_{+}+1, \ldots, m
\end{aligned}
$$

and continuous functions

$$
\begin{gathered}
U_{j}^{+}=U_{j,\left(\eta_{1}^{j}, \ldots, \eta_{m_{+}}^{j}\right)}^{+}:\left[0, \omega_{j}\right] \rightarrow \tilde{X} \\
U_{j}^{-}=U_{j,\left(\eta_{m_{+}+1}^{j}, \ldots, \eta_{m}^{j}\right)}^{-}: \quad\left[-\omega_{j-1}, 0\right] \rightarrow \tilde{X} .
\end{gathered}
$$

Moreover, if the variables $\eta_{1}^{j}, \ldots, \eta_{m}^{j}$ are fixed, the functions $U_{j}^{ \pm}$satisfy the following conditions a), b), c), d) and are uniquely determined by these:

a) $U_{j}^{+}$and $U_{j}^{-}$are classical solutions of $(6)$ on $\left(0, \omega_{i}\right)$ and $\left(-\omega_{i-1}, 0\right)$, respectively.

b) $U_{j}^{+}(0), U_{j}^{-}(0) \in B_{\delta_{*}}(H(0))$ with respect to the $\tilde{X}$-topology. Moreover, $U_{j}^{+}(0) \in \tilde{\Sigma}:=H(0)+\tilde{\mathcal{E}}_{+}^{s}(0)+\tilde{\mathcal{E}}_{-}^{u}(0)+\tilde{K}$ and $U_{j}^{-}(0) \in \tilde{\Sigma}$ and $U_{j}^{+}\left(\omega_{j}\right)=$ $U_{j+1}^{-}\left(-\omega_{j}\right)$.

c) $U_{j}^{+}(0)-U_{j}^{-}(0)=: \xi_{j}\left(\left\{\omega_{l}\right\}_{l \in \mathbb{Z}}, c, \eta_{1}^{j}, \ldots, \eta_{m}^{j}\right) \in \tilde{K}$.

d) $\sup _{\left[0, \omega_{i}\right]}\left\|U_{j}^{+}(s)-H(s)\right\|_{\tilde{X}} \leq \delta_{*}, \quad \sup _{\left[-\omega_{i-1}, 0\right]}\left\|U_{j}^{-}(s)-H(s)\right\|_{\tilde{X}} \leq \delta_{*}$.

We also have the property that

$$
\sup _{\left[0, \omega_{j}\right]}\left\|H(t)-U_{j}^{+}(t)\right\|_{\tilde{X}}+\sup _{\left[-\omega_{j-1}, 0\right]}\left\|H(t)-U_{j}^{-}(t)\right\|_{\tilde{X}} \rightarrow 0
$$

as $\omega_{j}, \omega_{j-1} \rightarrow \infty$. For fixed $\eta_{1}^{j}, \ldots, \eta_{m}^{j}$ and any $\tilde{C}>\omega_{*}$, the mapping

$$
\xi_{j}\left(\cdot, c, \eta_{1}^{j}, \ldots, \eta_{m}^{j}\right): l^{\infty}\left(\mathbb{Z},\left(\omega_{*}, \tilde{C}\right)\right) \rightarrow \tilde{X}
$$

is $C^{0}$ with respect to $\left\{\omega_{k}\right\}_{k \in \mathbb{Z}}$ if $c \in B_{\delta_{*}}\left(c_{*}\right)$ is fixed. Moreover, the function

$$
\xi_{j}\left(\left\{\omega_{k}\right\}_{k \in \mathbb{Z}}, \cdot, \eta_{1}^{j}, \ldots, \eta_{m}^{j}\right): B_{\delta_{*}}\left(c_{*}\right) \rightarrow \tilde{X}
$$


is $C^{2}$ for every fixed $\left\{\omega_{k}\right\}_{k \in \mathbb{Z}}, \omega_{k} \in\left(\omega_{*}, \infty\right) \cup\{\infty\}$ and associated $\eta_{1}^{j}, \ldots, \eta_{m}^{j}$. Finally, for every $j \in \mathbb{Z}$ and $C_{*}>\omega_{*}$ the mapping

$$
\left(\eta_{1}, \ldots, \eta_{m}\right) \mapsto \xi_{j}\left(\left\{\omega_{k}\right\}_{k \in \mathbb{Z}}, c,\left(\eta_{1}, \ldots, \eta_{m}\right)\right)
$$

is $C^{2}$ with respect to $\left(\eta_{1}, \ldots, \eta_{m}\right) \in \mathbb{R}^{m}$ for any fixed $c$ with $\left|c-c_{*}\right|<\delta_{*}$ and $\left\{\omega_{k}\right\}_{k \in \mathbb{Z}}$, if $\omega_{k}<C_{*}$ for all $k$.

The theorem states that upon choosing sufficiently large flight times $\omega_{j}$ for each $j \in \mathbb{Z}$, there exist solution segments $U_{i}$ on $\left[-\omega_{i}, \omega_{i}\right]$, composed by $U_{j}^{+}$and $U_{j+1}^{-}$, which may have jumps along an $(m+1)$-dimensional vector space $\tilde{K}$. The values $U_{j}^{+}(0), U_{j}^{-}(0)$ itself lie in $\tilde{\Sigma}$, which is nothing else but a Poincaré section (see also lemma 3 ). If all the jumps vanish (i.e. $\xi_{j}=0$ for all $j$ ), then a concatenation of the segments $U_{j}$ provides a globally defined solution of the equation (7) and in particular can be reinterpreted as a travelling wave solution of (1). In this respect we can regard the jump-functions $\xi_{j}$ as bifurcation equations, where the flight times $\left\{\omega_{k}\right\}_{k \in \mathbb{Z}}$, the "center" variables $\eta_{1}^{j}, \ldots, \eta_{m}^{j}$ and the travelling wave speed $c$ serve as parameters. The variables $\eta_{1}^{j}, \ldots, \eta_{m}^{j}$ originate from the fact that the steady state possesses an $m$-dimensional center eigenspace, and we will show where these parameters enter the analysis in lemma 5 and lemma 7 . Since also the dimension of the space $\tilde{K}$ incorporates the dimension $m$ of the center eigenspace, we can regard these center variables as "directions" for which we could not solve the bifurcation functions a priori by using the implicit function theorem. As a technical point, it then would be very desirable to have more than continuous dependence on the flight times $\left\{\omega_{k}\right\}_{k \in \mathbb{Z}}$ which in the case of hyperbolic steady states (i.e. $m=0$ ) has recently been proved in [19].

We should point out that using theorem 3 we are also able to detect globally defined solutions $U$, whose profile can be far away from the original profile of $H$ pointwisely. But every solution $U$ which can be constructed via theorem 3 is close to the homoclinic solution in the sense that its orbit has to be sufficiently close to the orbit of the primary homoclinic solution $H$; i.e. every point $U(\xi)$ is close to some point $H(\tilde{\xi})$ for some $\tilde{\xi} \in \mathbb{R}$. As an application, one can apply this reduction method to investigate the existence of multipulse solutions of (1) near the solitary wave. These are solutions whose profile resembles a concatenation of finitely many copies of the solitary wave profile and therefore its orbits with respect to the abstract setting of the travelling wave equation is close to the orbit of $H$. Let us also mention that hypothesis 2 is not necessary for theorem 3 to be true. In fact, if the intersection of $E_{+}^{c s}(0) \cap E_{-}^{u}(0)$ is $k$-dimensional for some $k \geq 2$ then theorem 3 holds verbally, where $\tilde{Z}$ now is $k$-dimensional.

There is an important feature that one can make use of when analysing the existence of periodic solutions near a homoclinic solution $H$. As one can easily show, these solutions are in one-to-one correspondence to the zero set of the bifurcation function $\xi_{1}(\omega, \omega, c)=0$ for some $\omega>0$ large enough and some travelling wave speed $c$ near the speed $c_{*}$ of the original solitary wave (where we have suppressed the parameter dependence on $\eta_{1}^{1}, \ldots, \eta_{m}^{1}$ for the moment). In fact, any zero of $\xi_{1}(\omega, \omega, c)=0$ induces a $2 \omega$-periodic solution of (16). The important aspect arises when the abstract equation (17) is reversible, which 
arises if the original lattice equation is time-reversible (typical examples are the Klein-Gordon, the Toda and Fermi-Pasta Ulam lattice, see [16, 17]). This is the case which will be treated in section 6 . In this case we will show in lemma 12 that $\xi$ takes only values in $\operatorname{Fix}(-\mathcal{R}) \cap \tilde{K}$, provided we have chosen $\tilde{K}$ to be $\mathcal{R}$-invariant, which we can do on account of the results in section 3.4. As a consequence, we do not really have to solve $m+1$ but typically only $\frac{m}{2}$ equations if $m$ is even. For example, if the homoclinic solution approaches a hyperbolic equilibrium (i.e. $m=0$ ) and is symmetric (i.e. intersects $\operatorname{Fix}(\mathcal{R})$ ) then we can actually choose a one-dimensional complement $\tilde{K}=\tilde{Z} \subset \operatorname{Fix}(\mathcal{R})$ and therefore $\xi_{1}\left(\omega, \omega, c_{*}\right)=0$ is automatically satisfied. This observation has been used in [17] to prove the existence of a one-parameter family of periodic solutions near the homoclinic solution in a reversible setting; a phenomenon which is typically referred to as the blue sky catastrophe.

But we can also make use of the reversibility in the framework of more complicated scenarios (as in the case of the Fermi-Pasta Ulam lattice in section $6)$, where $m=2$ and the reversibility allows us to reduce the number of bifurcation equations to just a single one (and hence from $3=m+1$ to 1 ). These observations show that one can make use of underlying structures, which often helps to reduce the number of bifurcation equations.

\subsection{The proof of the main result}

In this chapter we will prove our main result, theorem 3. We begin by giving an outline in the next section. There, we also will see where the parameters $\eta_{1}, \ldots, \eta_{m}$ enter.

\subsubsection{An outline of the proof}

In order to prove theorem 3, we will write a solution $U$ of $(17)$ in the form $U(t)=H(t)+V(t)$. Then $V$ solves the equation

$$
\dot{V}=\mathcal{A}(t) V(t)+\mathcal{G}(V(t), t, c),
$$

where we have set $\mathcal{G}(t, V, c):=\mathcal{F}(H(t)+V, c)-\mathcal{F}(H(t), c)-\mathcal{A}(t) V$ for $V \in \tilde{X}$. Hence, instead of proving theorem 3 we can alternatively show the next lemma:

\section{Lemma 4}

Fix constants $M_{*}, \gamma>0$ and a complement $\tilde{K}$ and for each $j \in \mathbb{Z}$ choose some positive integers $m_{+}^{j}, m_{-}^{j}$ with the property $m_{+}^{j}+m_{-}^{j}=m$. Then there exists constants $\omega_{*}>0$ and $\delta>0$ such that the following is true. Let $\left\{\omega_{j}\right\}_{j \in \mathbb{Z}}$ be sequence of flight times, $\omega_{j} \in\left(\omega_{*}, \infty\right) \cup\{\infty\}$. Then for every $j \in \mathbb{Z}$ there exist $m$ parameters $\eta_{1}^{j}, \ldots, \eta_{m}^{j}$ as in the statement of theorem 3 and additionally there exist classical solutions

$$
\begin{aligned}
& \bar{V}_{j}^{+}=\bar{V}_{j, \eta_{1}^{j}, \ldots, \eta_{m_{-}}^{j}}:\left[0, \omega_{j}\right] \rightarrow \tilde{X} \\
& \bar{V}_{j}^{-}=\bar{V}_{j, \eta_{1}^{j}, \ldots, \eta_{m_{+}}^{j}}^{-}:\left[-\omega_{j-1}, 0\right] \rightarrow \tilde{X}
\end{aligned}
$$


of $V^{\prime}(t)=\mathcal{A}(t) V(t)+\mathcal{G}(t, V(t))$. Once the parameters $\eta_{1}^{j}, \ldots, \eta_{m}^{j}$ are fixed, the solutions $\bar{V}_{j}^{-}, \bar{V}_{j}^{+}$are uniquely determined by satisfying the following properties I), II), III):

$$
\begin{aligned}
& \text { I) } \bar{V}_{j}^{+}\left(\omega_{j}\right)-\bar{V}_{j+1}^{-}\left(-\omega_{j}\right)=H\left(-\omega_{j}\right)-H\left(\omega_{j}\right) . \\
& \text { II) } \bar{V}_{j}^{ \pm}(0) \in\left\{\tilde{\mathcal{E}}_{+}^{s}(0)+\tilde{\mathcal{E}}_{-}^{u}(0)+\tilde{K}\right\} \cap B_{\delta}(0) \text { and } \bar{V}_{j}^{+}(0)-\bar{V}_{j}^{-}(0) \in \tilde{K} . \\
& \text { III) } \sup _{\left[0, \omega_{j}\right]}\left\|V_{j}^{+}(s)\right\|_{\tilde{X}}<\delta_{*}, \quad \sup _{\left[-\omega_{j-1}, 0\right]}\left\|V_{j}^{-}(s)\right\|_{\tilde{X}}<\delta_{*} .
\end{aligned}
$$

It is also true that

$$
\sup _{\left[0, \omega_{j}\right]}\left\|V_{+}^{j}(t)\right\|_{\tilde{X}}+\sup _{\left[-\omega_{j-1}, 0\right]}\left\|V_{-}^{j}(t)\right\|_{\tilde{X}} \rightarrow 0
$$

as $\omega_{j}, \omega_{j-1} \rightarrow \infty$. Moreover, define $\xi_{j}\left(\left\{\omega_{k}\right\}_{k \in \mathbb{Z}}, c,\left(\eta_{1}^{j}, \ldots, \eta_{m}^{j}\right)\right):=\bar{V}_{j}^{+}(0)-$ $\bar{V}_{j}^{-}(0)$. Then, $\xi_{j}$ satisfies all the properties which are listed at the end of theorem 3.

Let us now give an outline of how we intend to prove this lemma. We will construct $V_{i}^{+}$as a fixed point of the integral equation

$$
\begin{aligned}
V_{i}^{+}(t) & =\Phi_{+}^{s}(t, 0) V^{s,+}+\int_{0}^{t} \Phi_{+}^{s}(t, s) \mathcal{G}\left(V_{i}^{+}(s), s\right) d s \\
& +\Phi_{+}^{c u}\left(t, \omega_{i}\right) V^{c u,+}+\int_{\omega_{i}}^{t} \Phi_{+}^{c u}(t, s) \mathcal{G}\left(V_{i}^{+}(s), s\right) d s
\end{aligned}
$$

for suitable $V^{s,+} \in \tilde{\mathcal{E}}_{+}^{s}(0)$ and $V^{c u,+} \in \tilde{E}_{+}^{c u}\left(\omega_{i}\right)$, where we look for fixed points in the set $V_{i}^{+} \in B C^{\kappa,+, i}:=B C^{\kappa}\left(\left[0, \omega_{i}\right], \mathbb{R}^{N} \times C^{0}\left([-M, M], \mathbb{R}^{N}\right)\right)$ and some $\kappa>0$ small enough. Here, $B C^{\kappa,+, i}$ is equipped with the norm $|\cdot|_{\kappa}$, where for any $V \in B C^{\kappa,+, i}$ we have

$$
|V|_{\kappa}:=\sup _{\left[0, \omega_{i}\right]} e^{|\kappa||t|}|V(t)|_{\mathbb{R}^{N} \times C^{0}\left([-M, M], \mathbb{R}^{N}\right)}<\infty .
$$

We will show in the next sections that the right hand side of (21) in fact induces a contraction in $B C^{\kappa,+, i}$ which is uniform in $\omega_{i}>0$. Moreover, it has been shown in the proof of theorem 6 in [15] that any fixed point of (21) defines a weak solution of the equation (19). This weak solution is actually a classical solution for our special choice of $V^{s,+}$.

Similarly, we will construct $V_{i}^{-}$as fixed point of the equation

$$
\begin{aligned}
V_{i}^{-}(t) & =\Phi_{-}^{u}(t, 0) V^{u,-}+\int_{0}^{t} \Phi_{-}^{u}(t, s) \mathcal{G}\left(V_{i}^{-}(s), s\right) d s \\
& +\Phi_{-}^{c s}\left(t,-\omega_{i-1}\right) V^{c s,-}+\int_{-\omega_{i-1}}^{t} \Phi_{-}^{c s}(t, s) \mathcal{G}\left(V_{i}^{-}(s), s\right) d s
\end{aligned}
$$

for suitable $V^{u,-} \in \tilde{\mathcal{E}}_{-}^{u}(0)$ and $V^{c s,-} \in \tilde{E}_{-}^{c s}\left(-\omega_{i-1}\right)$, where we look for fixed points $V_{i}^{-} \in B C^{\kappa,-, i}:=B C^{\kappa}\left(\left[-\omega_{i-1}, 0\right], \mathbb{R}^{N} \times C^{0}\left([-M, M], \mathbb{R}^{N}\right)\right)$. Moreover, for given $V^{c u,+}, V^{c s,-}$ we will have to choose $V^{s,+}, V^{u,-}$ in order to ensure that

$$
U_{i}^{+}(0)-U_{i}^{-}(0)=V_{i}^{+}(0)-V_{i}^{-}(0) \in \tilde{K} .
$$


This will leave us finally to construct $V^{c s,-}, V^{c u,+}$ in order to guarantee

$$
U_{i}^{+}\left(\omega_{i}\right)=U_{i+1}^{-}\left(-\omega_{i}\right)
$$

which can translates into $V_{i}^{+}\left(\omega_{i}\right)-V_{i+1}^{-}\left(-\omega_{i}\right)=H\left(-\omega_{i}\right)+H\left(\omega_{i}\right)$. In this last step we will in fact show that $(24)$ can be achieved for a $m$-parameter family of values $V^{c s,-}, V^{c u,+}$. Hence, it is here where the parameters $\eta_{1}^{i}, \ldots, \eta_{m}^{i}$ enter. Technically, this stems from the $m$-dimensional intersection of

$$
\tilde{E}_{-}^{c s}\left(-\omega_{i}\right) \cap \tilde{E}_{+}^{c u}\left(\omega_{i}\right)
$$

for every $i \in \mathbb{Z}$ if $\omega_{i}>\omega_{*}$ and $\omega_{*}$ is large enough, which is proved in lemma 5 . Let us note that in the case of a hyperbolic steady state $(m=0)$ the intersection $(25)$ reduces to

$$
\tilde{E}_{-}^{s}\left(-\omega_{i}\right) \cap \tilde{E}_{+}^{u}\left(\omega_{i}\right)=\{0\}
$$

as has been shown in [17]. This shows that the $m$ additional parameters are a consequence from the absence of hyperbolicity at the asymptotic steady state; at least, if we work with the integral equation (21),(22). But only in this setting we are able to guarantee that the constructed fixed points $V_{i}^{ \pm}$are solutions of (19) and not just a modified equation, where the nonlinearity $\mathcal{G}$ is replaced by a modified nonlinearity $\mathcal{G}_{\text {mod }}$, which possesses a globally sufficient small Lipschitz-constant. This shows that we can think of the parameters $\eta_{1}^{i}, \ldots, \eta_{m}^{i}$ as parameters on the center eigenspace with respect to the linearization at the steady state for each $i \in \mathbb{Z}$.

\subsubsection{Proof of main result}

In order to construct solutions of (19) rigorously, we will carry out the program outlined in the previous section and therefore follow along the lines of the proof in the hyperbolic case $m=0$, see [17]. Let us first study the inhomogeneous linear equation

$$
\dot{V}_{i}^{ \pm}=\mathcal{A}(t) V_{i}^{ \pm}(t)+h_{i}^{ \pm}(t)
$$

$h_{i}=\left(h_{i}^{+}, h_{i}^{-}\right) \in X_{i-1, i}^{\kappa}$ where

$$
X_{i-1, i}^{\kappa}:=\left\{\left(h^{-}, h^{+}\right) \in B C^{\kappa, i,-} \times B C^{\kappa, i,+}\right\} .
$$

Preliminary results for identifying the center parameters

In order to identify the parameters $\eta_{1}^{i}, \ldots, \eta_{m}^{i}$ we first need the following lemma:

\section{Lemma 5}

Choose integers $m_{+}^{i}, m_{-}^{i}>0$ such that $m_{+}^{i}+m_{-}^{i}=m$ for all $i$ and write $\tilde{E}^{c}\left(\omega_{i}\right):=\tilde{E}_{-}^{c s}\left(-\omega_{i}\right) \cap \tilde{E}_{+}^{c u}\left(\omega_{i}\right)$. Then there exist and $\omega_{*}>0$ and subspaces

- $\bar{E}_{+}^{c u}\left(\omega_{i}\right) \subset \tilde{E}_{+}^{c u}\left(\omega_{i}\right)$,

- $\bar{E}_{-}^{c s}\left(-\omega_{i}\right) \subset \tilde{E}_{-}^{c s}\left(-\omega_{i}\right)$ 
which are closed in $\tilde{X}$ such that $\bar{E}_{+}^{c u}\left(\omega_{i}\right) \oplus \bar{E}_{-}^{c s}\left(-\omega_{i}\right)=\tilde{X}$ if $\omega_{i}>\omega_{*}$ and

- $\operatorname{dim}\left(\bar{E}_{+}^{c u}\left(\omega_{i}\right) \cap \tilde{E}^{c}\left(\omega_{i}\right)\right)=m_{-}$and

- $\operatorname{dim}\left(\bar{E}_{-}^{c s}\left(-\omega_{i}\right) \cap \tilde{E}^{c}\left(\omega_{i}\right)\right)=m_{+}$,

where the space $\tilde{E}^{c}\left(\omega_{i}\right)$ is m-dimensional.

\section{Proof}

We construct only the space $\bar{E}_{+}^{c u}\left(\omega_{i}\right)$, closed in $\tilde{X}$. In order to do that, let us denote by

$$
\tilde{E}_{+}^{u u}\left(\omega_{i}\right):=\operatorname{Rg}\left(\left.\Phi_{+}^{u u}\left(\omega_{i}, \omega_{i}\right)\right|_{\tilde{X}}\right)
$$

the strong unstable space with respect to a center stable dichotomy of the linear equation $\dot{V}(t)=\mathcal{A}(t) V(t)$ on $\mathbb{R}_{+}$. Then by the results in [16] it follows that $\tilde{E}_{+}^{u u}\left(\omega_{i}\right) \subset \tilde{E}_{+}^{c u}\left(\omega_{i}\right)$ has codimension $m$ in $\tilde{E}_{+}^{c u}\left(\omega_{i}\right)$, see also lemma 2. Now choose $m$ linear independent vectors $b_{1}, \ldots, b_{m_{-}}, b_{m_{-}+1}, \ldots, b_{m} \in \tilde{X}$ in $\tilde{E}^{c}\left(\omega_{i}\right)$ (which we assume to be $m$-dimensional for the moment) and define

$$
\bar{E}_{+}^{c u}\left(\omega_{i}\right):=\tilde{E}_{+}^{u u}\left(\omega_{i}\right)+\operatorname{span}\left\langle b_{1}, \ldots, b_{m_{-}}\right\rangle .
$$

The fact that $\bar{E}_{+}^{c u}\left(\omega_{i}\right)$ is closed now follows as in the proof of lemma 6.2 in [14]. $\bar{E}_{-}^{c s}\left(-\omega_{i}\right)$ can be constructed analogously. Finally, let us address the fact that $\tilde{E}^{c}\left(\omega_{i}\right)$ is $m$-dimensional. First note that

$$
\tilde{E}_{-}^{c s}\left(-\omega_{i}\right) \oplus \tilde{E}_{+}^{u u}\left(\omega_{i}\right)=\tilde{X}
$$

as follows by lemma 4 in [17]. Now we observe that the codimension of $\tilde{E}_{+}^{u u}\left(\omega_{i}\right)$ in $\tilde{E}_{+}^{c u}\left(\omega_{i}\right)$ is $m$ which shows all claims of the lemma.

\section{Definition 4}

The last lemma and its proof allow us to define the bounded projection $\bar{P}^{m_{+}}\left(\omega_{j}\right)$ : $\tilde{X} \rightarrow \tilde{X}$ with $m_{+}$-dimensional $\operatorname{Rg}\left(\bar{P}^{m_{+}}\left(\omega_{j}\right)\right)=\operatorname{span}\left\langle b_{m_{-}+1}, \ldots, b_{m}\right\rangle$ according to the decomposition of $\tilde{X}$ via

$$
\operatorname{span}\left\langle b_{1}, \ldots, b_{m_{-}}\right\rangle \oplus \operatorname{span}\left\langle b_{m_{-}+1}, \ldots, b_{m}\right\rangle \oplus \tilde{E}_{+}^{u u}\left(\omega_{j}\right) \oplus \tilde{E}_{+}^{s}\left(\omega_{j}\right) .
$$

Similarly, we define the bounded projection $\bar{P}^{m_{-}}\left(-\omega_{j}\right): \tilde{X} \rightarrow \tilde{X}$ with $m_{--}$ dimensional $\operatorname{Rg}\left(\bar{P}^{m_{-}}\left(-\omega_{j}\right)\right)=\operatorname{span}\left\langle\tilde{b}_{m_{+}+1}, \ldots, \tilde{b}_{m}\right\rangle$ with respect to the decomposition

$$
\operatorname{span}\left\langle\tilde{b}_{m_{+}+1}, \ldots, \tilde{b}_{m}\right\rangle \oplus \tilde{E}_{-}^{s s}\left(-\omega_{j}\right) \oplus \tilde{E}_{-}^{u}\left(-\omega_{j}\right) \oplus \operatorname{span}\left\langle\tilde{b}_{1}, \ldots, \tilde{b}_{m_{+}}\right\rangle .
$$

An important case arises, if the original equation is reversible with respect to some reverser $\mathcal{R}$, see section 3.3 for a definition of reversibility and the class of admissible reversers. Then, we have the following result.

\section{Lemma 6}

Assume that the original equation is $\mathcal{R}$-invariant in the sense of (14). Then, for any $\omega>0$, the space $\tilde{E}^{c}\left(\omega_{i}\right):=\tilde{E}_{-}^{c s}\left(-\omega_{i}\right) \cap \tilde{E}_{+}^{c u}\left(\omega_{i}\right)$ can be chosen $\mathcal{R}$-invariant. 


\section{Proof}

First of all note that for any closed space $E \subset Y$ with $E \oplus E_{+}^{s}(0)=Y$ there exists a center unstable dichotomy on $\mathbb{R}_{+}$with associated operators $\Phi_{+}^{c u}, \Phi_{+}^{s}$ such that $\operatorname{Rg}\left(\Phi_{+}^{c u}(0,0)\right)=E$, see for example [28]. We now make the particular choice

$$
E:=\mathcal{E}_{-}^{u}(0)+\tilde{Z}+\tilde{K}^{c} \subset Y,
$$

where $\tilde{K}^{c}$ is $m$-dimensional and defined in the statement of theorem 3 and $\mathcal{E}_{-}^{u}(0) \subset E_{-}^{u}(0)$ denotes a complement of $\mathcal{F}\left(H(0), c_{*}\right) \in E_{-}^{u}(0)$. We note that $\mathcal{E}_{-}^{u}(0) \oplus E_{+}^{s}(0)$ has codimension $m+1$ in $Y$, see lemma 5 in [16] (which shows that $\tilde{K}^{c}$ is indeed $m$-dimensional). For a rigorous proof that the right hand side of (27) now defines a closed space in $Y$ we refer to lemma 6.2 and remark 6.1 in [14]. Taking into account the results in [16] and in particular the ideas in the proof of lemma 7 there, we then also have the identity

$$
\operatorname{Rg}\left(\left.\Phi_{+}^{c u}(0,0)\right|_{\tilde{X}}\right)=E \cap \tilde{X}=\tilde{\mathcal{E}}_{-}^{u}(0)+\tilde{Z}+\tilde{K}^{c}
$$

and $E \cap \tilde{X}$ is closed with respect to the $\tilde{X}$-norm. With the help of the so defined operators $\Phi_{+}^{c u}, \Phi_{+}^{s}$, we can define a center stable dichotomy on $\mathbb{R}_{-}$with associated solution operators $\Phi_{-}^{u}(t, s), t \leq s \leq 0$ and $\Phi_{-}^{c s}(t, s), s \leq t \leq 0$ via

$$
\begin{aligned}
& \Phi_{-}^{c s}(t, s):=\mathcal{R} \Phi_{+}^{c u}(-t,-s) \mathcal{R} \\
& \Phi_{-}^{u}(t, s):=\mathcal{R} \Phi_{+}^{s}(-t,-s) \mathcal{R} .
\end{aligned}
$$

Then we have $\left.\left.\operatorname{Rg}\left(\Phi_{-}^{c s}(0,0)\right)\right|_{\tilde{X}}\right)=\tilde{\mathcal{E}}_{+}^{s}(0)+\tilde{K}$ provided the space $\tilde{K}$ is $\mathcal{R}$ invariant. It is then true that

$$
\mathcal{R}\left[\tilde{E}_{+}^{c u}(\omega)\right]=\tilde{E}_{-}^{c s}(-\omega)
$$

for any $\omega \geq 0$. In order to see that (28) defines a center stable dichotomy on

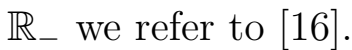

Finally, let us show that $\tilde{E}^{c}\left(\omega_{i}\right)$ is $\mathcal{R}$-invariant. Let us therefore choose any $V \in \tilde{E}^{c}$. Then $V \in \tilde{E}_{-}^{c s}\left(-\omega_{i}\right)$ which means that $\mathcal{R} V \in \tilde{E}_{+}^{c u}\left(\omega_{i}\right)$. Similarly, $V \in \tilde{E}_{+}^{c u}\left(\omega_{i}\right)$ implies that $\mathcal{R} V \in \tilde{E}_{-}^{c s}\left(-\omega_{i}\right)$ which shows the claim.

\section{Constructions of solutions for the linear inhomogeneous equation}

For the rest of the section we now choose some positive integers $m_{-}, m_{+}$ with associated subspaces $\bar{E}_{+}^{c u}\left(\omega_{i}\right), \bar{E}_{-}^{c s}\left(\omega_{i}\right)$ satisfying the properties of the last lemma. Hence, we can write any $a^{+} \in \tilde{E}_{+}^{c u}\left(\omega_{j}\right)$ and $a^{-} \in \tilde{E}_{-}^{c s}\left(-\omega_{j}\right)$ for any $\omega_{j}>0$ large enough in the form $a^{ \pm}=\left(\tilde{a}^{ \pm}, \eta^{ \pm}\right)$, where $\tilde{a}^{+} \in \bar{E}_{+}^{c u}\left(\omega_{j}\right)$, $\tilde{a}^{-} \in \bar{E}_{-}^{c s}\left(-\omega_{j}\right)$ and

$$
\eta^{+} \in \operatorname{Rg}\left(\bar{P}^{m_{+}}\left(\omega_{j}\right)\right), \quad \eta^{-} \in \operatorname{Rg}\left(\bar{P}^{m_{-}}\left(-\omega_{j}\right)\right),
$$

where the projections have been defined in definition 4 . We now choose a $(1+m)$-dimensional space $\tilde{K}$ as in the statement of theorem 3 . Then we have the following lemma: 


\section{Lemma 7}

Let $\omega_{i}>0, h_{i} \in X_{i-1, i}^{\kappa}$ for all $i$ and choose

$$
a_{i}=\left(a_{i}^{+}, a_{i}^{-}\right)=\left(\left(\tilde{a}_{i}^{+}, \eta_{i}^{+}\right),\left(\tilde{a}_{i}^{-}, \eta_{i}^{-}\right)\right) \in \tilde{E}_{+}^{c u}\left(\omega_{i}\right) \times \tilde{E}_{-}^{c s}\left(-\omega_{i-1}\right) .
$$

Then if $\omega_{i}>\omega_{*}$ for all $i$ and $\omega_{*}>0$ is large enough, there exist unique classical solutions of $\dot{V}_{i}^{ \pm}=\mathcal{A} V_{i}^{ \pm}+h_{i}^{ \pm}$such that $V_{i} \in X_{i-1, i}^{\kappa}$ satisfies

- $P^{c u,+}\left(\omega_{i}\right) V_{i}^{+}\left(\omega_{i}\right)=a_{i}^{+}, P^{c s,-}\left(-\omega_{i-1}\right) V_{i}^{-}\left(-\omega_{i-1}\right)=a_{i}^{-}$and

- $\bar{P}^{m_{+}}\left(\omega_{i}\right) V_{i}^{+}\left(\omega_{i}\right)=\eta_{i}^{+}, \bar{P}^{m_{-}}\left(\omega_{i-1}\right) V_{i}^{-}\left(-\omega_{i-1}\right)=\eta_{i}^{-}$.

Finally, it is true that $V_{i}^{ \pm}(0) \in\left\{\tilde{\mathcal{E}}_{+}^{s}(0)+\tilde{\mathcal{E}}_{-}^{u}(0)+\tilde{K}\right\}$ and $V_{i}^{+}(0)-V_{i}^{-}(0) \in \tilde{K}$ for all $i \in \mathbb{Z}$, where $\tilde{K}$ has been defined in theorem 3 .

The proof of this lemma completely parallels the proof in [17] for the case $m=0$ and we therefore omit it. From now on we want to denote the solutions obtained in the last lemma by

$$
\bar{V}_{i}^{+}=\bar{V}_{i}^{+}\left(a_{i}^{+}, h_{i}^{+}, \omega_{j}\right), \quad \bar{V}_{i}^{-}=\bar{V}_{i}^{-}\left(a_{i}^{-}, h_{i}^{-}, \omega_{j-1}\right) .
$$

\section{Lemma 8}

The function $\bar{V}_{j}(t)=\bar{V}_{j}^{+}(t)$ for $t \geq 0$ and $\bar{V}_{j}(t):=\bar{V}_{j}^{-}(t)$ for $t<0$ has the following properties.

a) Let $t \in\left[-\omega_{j-1}, \omega_{j}\right]$. Then there are $\kappa, \delta, \alpha>0$ with $0<\delta<\kappa<\alpha$ and there exists a $j$-independent constant $C$ such that

$$
\begin{aligned}
\left|\bar{V}_{j}(t)\right|_{\tilde{X}} \leq C\left(e^{-\kappa|t|}\left\|h_{j}\right\|_{\kappa}+e^{\delta\left|\omega_{j}-t\right|}\left\|a_{j}^{+}\right\|_{\tilde{X}}+e^{-\alpha|t|}\left\|a_{j}^{-}\right\|_{\tilde{X}}\right), \quad t \geq 0 \\
\left|\bar{V}_{j}(t)\right|_{\tilde{X}} \leq C\left(e^{-\kappa|t|}\left\|h_{j}\right\|_{\kappa}+e^{\delta\left|\omega_{j-1}-t\right|}\left\|a_{j}^{-}\right\|_{\tilde{X}}+e^{-\alpha|t|}\left\|a_{j}^{+}\right\|_{\tilde{X}}\right), \quad t<0
\end{aligned}
$$

b) If $\omega_{j}>\omega_{*}$ for all $j$ and $\omega_{*}$ is large enough, then

$$
\begin{array}{r}
\left|P_{-}^{u}\left(-\omega_{j}\right) \bar{V}_{j+1}^{-}\left(-\omega_{j}\right)\right|_{\tilde{X}}+\left|P_{+}^{s}\left(\omega_{j}\right) \bar{V}_{j}^{+}\left(\omega_{j}\right)\right|_{\tilde{X}} \leq \\
C e^{-\kappa\left|\omega_{j}\right|}\left(\left\|h_{j}\right\|_{\kappa}+\max \left\{\left\|a_{j}^{+}\right\|_{\tilde{X}},\left\|a_{j}^{-}\right\|_{\tilde{X}}\right\} \|\right) .
\end{array}
$$

\section{Proof}

Let us first prove the claim a) where we only consider the case $t \in\left[0, \omega_{j}\right]$. Choose some $\delta, \kappa$ with $0<\delta<\kappa<\alpha$, where $\alpha>0$ denotes the exponentialdecay rate of the solution operator $\Phi_{+}^{s}$ and $\Phi_{-}^{u}$, i.e.

$$
\begin{aligned}
\left\|\Phi_{+}^{s}(t, s)\right\|_{L(\tilde{X}, \tilde{X})} & \leq C e^{-\alpha|t-s|}, & & 0 \leq s \leq t \\
\left\|\Phi_{-}^{u}(t, s)\right\|_{L(\tilde{X}, \tilde{X})} & \leq C e^{-\alpha|t-s|}, & & t \leq s \leq 0
\end{aligned}
$$

for some $C>0$. Note that $\alpha<\alpha_{*}$, where $\alpha_{*}$ denotes the decay rate of $H$, see hypothesis 1 . Then

$$
\begin{aligned}
V_{j}^{+}(t) & =\Phi_{+}^{s}(t, 0) V^{s,+}+\int_{0}^{t} \Phi_{+}^{s}(t, s) h_{j}^{+}(s) d s \\
& +\Phi_{+}^{c u}\left(t, \omega_{j}\right) a_{j}^{+}+\int_{\omega_{j}}^{t} \Phi_{+}^{c u}(t, s) h_{+}^{j}(s) d s
\end{aligned}
$$


for some specific value $V^{s,+}=V^{s,+}\left(h_{j}, a_{j}^{-}\right)$such that

$$
\left\|V^{s,+}\right\|_{\tilde{X}} \leq M \cdot e^{-\alpha \omega_{j-1}}\left\|a_{j}^{-}\right\|_{\tilde{X}}+M\left\|h_{j}\right\|_{\kappa} .
$$

In fact, this follows for the explicit choice of $V^{s,+}$ which immediately leads to (30), see the proof of lemma 8 in [17] or [22]. We can therefore compute

$$
\begin{aligned}
\left\|V_{j}^{+}(t)\right\|_{\tilde{X}} & \leq M e^{-\alpha|t|}\left\|V^{s,+}\right\|_{\tilde{X}}+\int_{0}^{t} M e^{-\alpha(t-s)} \cdot e^{-\kappa s}\left\|h_{j}\right\|_{\kappa} d s \\
& +M e^{\delta\left(\omega_{j}-t\right)}\left\|a_{j}^{+}\right\|_{\tilde{X}}+\int_{\omega_{j}}^{t} M e^{\delta(t-s)} e^{-\kappa s}\left\|h_{j}\right\|_{\kappa} d s \\
& \leq M e^{-\alpha|t|}\left\|V^{s,+}\right\|_{\tilde{X}}+\tilde{M} e^{-\kappa t}\left\|h_{j}\right\|_{\kappa}+M e^{\delta\left(\omega_{j}-t\right)}\left\|a_{j}^{+}\right\|_{\tilde{X}}
\end{aligned}
$$

from which a) follows together with (30).

Let us now explain why the property b) is true and let us again consider only $\bar{V}_{j}^{+}$. Since the values after applying the linear map $\Phi_{+}^{c u}\left(\omega_{j}, \omega_{j}\right)$ lie in the kernel of $P_{+}^{s}\left(\omega_{j}\right)$, we see from (29) that after applying $P_{+}^{s}\left(\omega_{j}\right)$ to the right hand side of (29) there only remain terms which decay exponentially as $\omega_{*} \rightarrow \infty$ and hence $\omega_{j} \rightarrow \infty$. Note that also in front of the term $V_{+}^{s}$ we have the exponentially decaying term $\Phi_{+}^{s}\left(\omega_{j}, 0\right)$ and therefore b) in the upper lemma is proves since one can estimate the term $P_{-}^{u}\left(-\omega_{j}\right) \bar{V}_{j}^{-}\left(-\omega_{j}\right)$ completely analogously.

\section{Lemma 9}

There exists an $\omega_{*}>0$ and a $\kappa>0$ such that for all $\omega_{j}>\omega_{*}$ and all $h_{j} \in X_{j, j-1}^{\kappa}$ the equation $\dot{V}_{j}^{ \pm}=\mathcal{A} V_{j}^{ \pm}+h_{j}^{ \pm}$possesses a solution $V_{j}=V_{j}\left(h_{j}, a_{j}\right) \in X_{j-1, j}^{\kappa}$ satisfying the following property: Write $a_{j}^{ \pm}=\left(\tilde{a}_{j}^{ \pm}, \eta_{j}^{ \pm}\right)$, then

$$
\begin{aligned}
\tilde{a}_{j}^{+}-\tilde{a}_{j+1}^{-} & =\left[H\left(-\omega_{j}\right)-H\left(\omega_{j}\right)\right] \\
& +P_{-}^{u}\left(-\omega_{j}\right) V_{j+1}^{-}\left(-\omega_{j}\right)-P_{+}^{s}\left(\omega_{j}\right) V_{j}^{+}\left(\omega_{j}\right)-\eta_{j}^{+}+\eta_{j+1}^{-} .
\end{aligned}
$$

Moreover, if there are elements $b_{i}^{ \pm}=\left(\tilde{b}_{i}^{ \pm}, \eta_{i}^{ \pm}\right)$satisfying (31) such that $V_{i}=$ $V_{i}\left(h_{i}, b_{i}\right) \in X_{i-1, i}^{\kappa}$, then $\tilde{b}_{i}^{+}=\tilde{a}_{i}^{+}$and $\tilde{b}_{i}^{-}=\tilde{a}_{i}^{-}$. Hence, $\tilde{a}_{j}^{ \pm}$only depends on $h_{j}, h_{j+1}$ and we have the estimate

$$
\begin{aligned}
\left\|\tilde{a}_{j}^{+}\right\|_{\tilde{X}}+\left\|\tilde{a}_{j+1}^{-}\right\|_{\tilde{X}} & \leq C e^{-\kappa \omega_{j}} \max \left\{\left\|h_{j}\right\|_{\kappa},\left\|h_{j+1}\right\|_{\kappa}\right\} \\
& +C\left(\left|\eta_{j}^{+}\right|+\left|\eta_{j+1}^{-}\right|\right)+C\left[\left|H\left(\omega_{j}\right)-H\left(\omega_{j}\right)\right|\right]
\end{aligned}
$$

for some $j$-independent constant $C$.

\section{Proof}

Only for this proof we need to define another projection which we denote by $\bar{P}_{j}: \tilde{X} \rightarrow \tilde{X}$ the bounded projection with range $\bar{E}_{-}^{c s}\left(-\omega_{j}\right)$ and kernel $\bar{E}_{+}^{c u}\left(\omega_{j}\right)$ for $\omega_{j-1}, \omega_{j}>\omega_{*}$ and $\omega_{*}$ large enough. Then $U_{j}^{+}\left(\omega_{j}\right)=U_{j+1}^{-}\left(-\omega_{j}\right)$ is equivalent to

$$
\begin{aligned}
{\left[P_{+}^{s}\left(\omega_{j}\right)+P_{+}^{c u}\left(\omega_{j}\right)\right] V_{j}^{+}\left(\omega_{j}\right) } & -\left[P_{-}^{u}\left(-\omega_{j}\right)+P_{-}^{c s}\left(-\omega_{j}\right)\right] V_{j+1}^{-}\left(-\omega_{j}\right) \\
& =H\left(-\omega_{j}\right)-H\left(\omega_{j}\right)
\end{aligned}
$$


which can be written in the form (31). Since $\tilde{a}_{j+1}^{-} \in \bar{E}_{-}^{c s}\left(-\omega_{j}\right)=\operatorname{Rg}\left(\bar{P}_{j}\right)$ and $\tilde{a}_{j}^{+} \in \bar{E}_{+}^{c u}\left(\omega_{j}\right)=\operatorname{Rg}\left(i d-\bar{P}_{j}\right)$, the identity (31) is equivalent to

$$
\begin{aligned}
\tilde{a}_{j+1}^{-} & =\bar{P}_{j}\left\{H\left(-\omega_{j}\right)-H\left(\omega_{j}\right)+P_{-}^{u}\left(-\omega_{j}\right) V_{j+1}^{-}\left(-\omega_{j}\right)\right\} \\
& +\bar{P}_{j}\left\{-P_{+}^{s}\left(\omega_{j}\right) V_{j}^{+}\left(\omega_{j}\right)-\eta_{j}^{+}+\eta_{j+1}^{-}\right\} \\
\tilde{a}_{j}^{+} & =\left(i d-\bar{P}_{j}\right)\left\{H\left(-\omega_{j}\right)-H\left(\omega_{j}\right)+P_{-}^{u}\left(-\omega_{j}\right) V_{j+1}^{-}\left(-\omega_{j}\right)\right\} \\
& +\left(i d-\bar{P}_{j}\right)\left\{-P_{+}^{s}\left(\omega_{j}\right) V_{j}^{+}\left(\omega_{j}\right)-\eta_{j}^{+}+\eta_{j+1}^{-}\right\} .
\end{aligned}
$$

In a more compact notation, (33) takes the form

$$
\left(\begin{array}{c}
\tilde{a}_{j+1}^{-} \\
\tilde{a}_{j}^{+}
\end{array}\right)=\mathcal{L}_{1}\left(\begin{array}{c}
\tilde{a}_{j+1}^{-} \\
\tilde{a}_{j}^{+}
\end{array}\right)+\mathcal{L}_{2}\left[H\left(-\omega_{j}\right)-H\left(\omega_{j}\right)\right]+\mathcal{L}_{3}\left(\begin{array}{c}
h_{j+1} \\
h_{j}
\end{array}\right)
$$

where all the operators $\mathcal{L}_{i}, i=1,2,3$, are linear, and the bounded map $\mathcal{L}_{1}$ : $L\left(\bar{E}_{-}^{c s}\left(-\omega_{j}\right) \times \bar{E}_{+}^{c u}\left(\omega_{j}\right)\right)$ has the property that

$$
\left\|\mathcal{L}_{1}\right\|_{L\left(\bar{E}_{-}^{c s}\left(-\omega_{j}\right) \times \bar{E}_{+}^{c u}\left(\omega_{j}\right)\right)} \rightarrow 0
$$

on account of property $b)$ of lemma 8 . This shows that the operator $\left(i d-\mathcal{L}_{1}\right) \in$ $L\left(\bar{E}_{-}^{c s}\left(-\omega_{j}\right) \times \bar{E}_{+}^{c u}\left(\omega_{j}\right)\right)$ is boundedly invertible and we can solve (34) for $\left(\tilde{a}_{j+1}^{-}, \tilde{a}_{j}^{+}\right)$in terms of $\eta_{j}^{ \pm}, \eta_{j+1}^{ \pm}$and $h_{j}^{+}, h_{j+1}^{-}$. Finally, the estimate (32) follows once more from property $b$ ) of lemma 8.

Note that this lemma allows us also to include the case of flight times $\left\{\omega_{l}\right\}_{l \in \mathbb{Z}}$ where $\omega_{k}=\infty$ for $k \in \mathcal{I}$ and some arbitrary subset $\mathcal{I} \subset \mathbb{Z}$. In fact, for these values of $k$ we choose $\tilde{a}_{k}^{+}=\tilde{a}_{k+1}^{-}=\eta_{k}^{+}=\eta_{k+1}^{-}=0$ and we can define the functions $V_{j}^{+}$via (29) and $V_{j}^{-}$accordingly.

\subsection{The nonlinear equation}

We are now ready to deal with the nonlinear equation

$$
\dot{V}_{j}^{ \pm}(t)=\mathcal{A}(t) V_{j}^{ \pm}(t)+\mathcal{G}\left(V_{j}^{ \pm}(t), t, c\right) .
$$

First we can choose a cut-off function $\chi_{\rho}:(-\rho, \rho) \rightarrow \mathbb{R}$ for every $\rho>0$, which has compact support in $(-\rho, \rho)$ and $\left.\chi\right|_{[-\rho / 2, \rho / 2]} \equiv 1$. Let us now consider the modified nonlinearity

$$
\mathcal{G}_{\text {mod }}(V, t, c):=\chi_{\rho}\left(\|V\|_{\tilde{X}}\right) \cdot \mathcal{G}(V, t, c)
$$

for any $V \in \tilde{X}$ and $\rho>0$. Then $\mathcal{G}_{\text {mod }}(\cdot, t, c): \tilde{X} \rightarrow \tilde{X}$ is Lipschitz-continuous and the Lipschitz-constant can be chosen to approach 0 as $\rho \searrow 0$. For given $c \approx c_{*}$, admissible flight times $\left\{\omega_{k}\right\}_{k \in \mathbb{Z}}, \omega_{k} \in\left(\omega_{*}, \infty\right) \cup\{\infty\}$ for all $k$, and $\left\{\eta_{l}^{ \pm}\right\}_{l \in \mathbb{Z}}$ we now want to construct a unique fixed point of the map

$$
\begin{aligned}
l: \oplus_{j \in \mathbb{Z}} X_{j, j-1}^{\kappa} & \rightarrow \oplus_{j \in \mathbb{Z}} X_{j, j-1}^{\kappa} \\
l:\left\{\left(V_{j}^{+}, V_{j}^{-}\right)\right\}_{j \in \mathbb{Z}} & \mapsto\left\{\left(\bar{V}_{j}^{+}, \bar{V}_{j}^{-}\right)\right\}_{j \in \mathbb{Z}} .
\end{aligned}
$$


Here,

$$
\bar{V}_{j}^{+}=\bar{V}_{j}^{+}\left(\tilde{a}_{j}^{+}, \mathcal{G}_{\text {mod }}\left(V_{j}^{+}, \cdot, c\right), \eta_{j}^{+}\right) \in B C^{\kappa}\left(\left[0, \omega_{j}\right], \tilde{X}\right)
$$

and $\tilde{a}_{j}^{+}$is uniquely determined by $\mathcal{G}\left(V_{j}^{+}(\cdot), \cdot, c\right), \mathcal{G}\left(V_{j+1}^{-}(\cdot), \cdot, c\right)$ and $\eta_{j}^{+}, \eta_{j+1}^{-}$on account of lemma 9. Moreover,

$$
\bar{V}_{j}^{-}=\bar{V}_{j}^{-}\left(\tilde{a}_{j}^{-}, \mathcal{G}_{\text {mod }}\left(V_{j}^{-}, \cdot, c\right), \eta_{j}^{-}\right) \in B C^{\kappa}\left(\left[-\omega_{j-1}, 0\right], \tilde{X}\right)
$$

and $\tilde{a}_{j}^{-}$is uniquely determined by $\mathcal{G}\left(V_{j-1}^{+}(\cdot), \cdot, c\right), \mathcal{G}\left(V_{j}^{-}(\cdot), \cdot, c\right)$ and $\eta_{j-1}^{+}, \eta_{j}^{-}$. We can now prove the following lemma, which proves lemma 4 . For the statement of the lemma, we remind the reader that the projections $\bar{P}^{m_{-}}\left(-\omega_{j}\right), \bar{P}^{m_{+}}\left(\omega_{j}\right)$ have been defined in definition 4 .

\section{Lemma 10}

There exists an $\omega_{*}>0$ and a $\kappa>0$ such that the following holds. For any given sequence of flight times $\left\{\omega_{l}\right\}_{l \in \mathbb{Z}}$ such that $\omega_{j} \in\left(\omega_{*}, \infty\right) \cup\{\infty\}$ for all $j$, and for every sequence $\left\{\eta_{j}^{ \pm}\right\}$with $\eta_{j}^{+} \in R g\left(\bar{P}^{m_{+}}\left(\omega_{j}\right)\right)$ and $\eta_{j+1}^{-} \in R g\left(\bar{P}^{m_{-}}\left(-\omega_{j}\right)\right)$ there is a unique fixed point

$$
\left\{\left(V_{j}^{+}, V_{j}^{-}\right)\right\} \in X_{j, j-1}^{\kappa}
$$

of the map $l$ defined in (36) for some $\rho>0$ small enough. This fixed point satisfies

$$
\bar{P}^{m_{+}}\left(\omega_{j}\right) V_{j}^{+}\left(\omega_{j}\right)=\eta_{j}^{+}, \quad \bar{P}^{m_{-}}\left(-\omega_{j}\right) V_{j+1}^{-}\left(-\omega_{j}\right)=\eta_{j}^{-}
$$

for all $j \in \mathbb{Z}$ and additionally we have the following properties:

A) For every $\left\{\omega_{j}\right\}_{j \in \mathbb{Z}}$, every $\gamma>0$ and every $M_{*}>0$ choose a sequence $\left\{\eta_{j}^{ \pm}\right\}$such that $\eta_{j}^{+}=\eta_{j}^{+}\left(\omega_{j}\right)$ and $\eta_{j}^{-}=\eta_{j}^{-}\left(\omega_{j-1}\right)$ have the property that

$$
\left|\eta_{j}^{+}\right| \leq M_{*} e^{-\gamma \omega_{j}}, \quad\left|\eta_{j}^{-}\right| \leq M_{*} e^{-\gamma \omega_{j-1}}
$$

where we have set $\eta_{j}^{+}=0$ if $\omega_{j}=\infty$ and where we have set $\eta_{j}^{-}=0$ if $\omega_{j-1}=\infty$. Then $l$ is a uniform contraction with respect to $\left\{\omega_{j}\right\}_{j \in \mathbb{Z}}$ and $\left\{\eta_{j}^{ \pm}\right\}$, if $\kappa$ is chosen even smaller if necessary to assure that $2 \kappa<\gamma$.

B) The fixed point $\left\{\left(V_{j}^{-}, V_{j}^{+}\right)\right\}_{j \in \mathbb{Z}}$ satisfies the properties I), II), III) and (20) of lemma 4.

C) Choose some $C>\omega_{*}$. Then, if $\left\{\eta_{j}^{ \pm}\right\}_{j \in \mathbb{Z}}$ and $c$ are fixed, the contraction $l$ (and therefore the fixed point $\left.\left\{\left(V_{j}^{-}, V_{j}^{+}\right)\right\}_{j \in \mathbb{Z}}\right)$ depends continuously on $\left\{\omega_{k}\right\}_{k \in \mathbb{Z}} \in l^{\infty}\left(\mathbb{Z},\left(\omega_{*}, C\right)\right)$.

D) If $\left\{\omega_{k}\right\}_{k \in \mathbb{Z}} \in l^{\infty}\left(\mathbb{Z},\left(\omega_{*}, \infty\right)\right)$ and $c$ are fixed, then $l$ depends $C^{2}$ on $\left\{\left(\eta_{j}^{+}, \eta_{j}^{-}\right)\right\}_{j \in \mathbb{Z}} \in l^{\infty}\left(\mathbb{Z}, \operatorname{Rg}\left(\bar{P}^{m_{+}}\left(\omega_{j}\right)\right) \times \operatorname{Rg}\left(\bar{P}^{m_{-}}\left(\omega_{j-1}\right)\right)\right)$.

E) If $\left\{\omega_{k}\right\}_{k \in \mathbb{Z}}, \omega_{k} \in\left(\omega_{*}, \infty\right) \cup\{\infty\}$ and the associated $\left\{\eta_{j}^{ \pm}\right\}$are fixed, then $l$ depends $C^{2}$ on $c \in B_{\delta_{*}}\left(c_{*}\right)$ for some sufficiently small $\delta_{*}>0$. 


\section{Proof}

Let us first show that $l$ is well-defined regarded as a map which takes values in $\oplus_{j \in \mathbb{Z}} X_{j, j-1}^{\kappa}$ and uniformly bounded with respect to $\left\{\omega_{k}\right\}_{k \in \mathbb{Z}}$. Let us only treat the case $t \in\left[0, \omega_{j}\right]$. Then using condition $a$ ) of lemma 8 we have the estimate

$$
\left.e^{\kappa|t|}\left|\bar{V}_{j}(t)\right|_{\tilde{X}} \leq C\left(\left\|\mathcal{G}\left(V_{j}^{ \pm}(\cdot), \cdot\right)_{j}\right\|_{\kappa}+e^{\delta\left|\omega_{j}-t\right|+\kappa|t|}\left\|a_{j}^{+}\right\|_{\tilde{X}}+e^{-\alpha t+\kappa t}\left\|a_{j}^{-}\right\|_{\tilde{X}}\right\}\right) .
$$

Taking into account the estimate (32) of lemma 9 , we see that $a_{j}^{+}$can be estimated in terms of

$$
\begin{aligned}
\left\|a_{j}^{+}\right\|_{\tilde{X}} & \leq e^{-\kappa\left|\omega_{j}\right|}\left(\|\left(\mathcal{G}\left(V_{j}^{+}(\cdot), \cdot, c\right)\left\|_{\kappa}+\right\| \mathcal{G}\left(V_{j+1}^{-}(\cdot), \cdot, c\right) \|_{\kappa}\right)\right. \\
& +C\left(\left|\eta_{j}^{+}\right|+\left|\eta_{j+1}^{-}\right|\right)+C\left[\left|H\left(\omega_{j}\right)-H\left(-\omega_{j}\right)\right|\right],
\end{aligned}
$$

where we recall that $0<\delta<\kappa<\alpha$. A similar estimate holds for the term $a_{j}^{-}$. Therefore,

$$
\begin{aligned}
e^{\kappa|t|}\left|\bar{V}_{j}(t)\right|_{\tilde{X}} & \leq C\left[1+e^{\delta\left|\omega_{j}-t\right|+\kappa|t|-\kappa \omega_{j}}\right]\left(\|\left(\mathcal{G}\left(V_{j}^{+}(\cdot), \cdot, c\right)\left\|_{\kappa}+\right\| \mathcal{G}\left(V_{j+1}^{-}(\cdot), \cdot, c\right) \|_{\kappa}\right)\right. \\
& +C e^{\kappa \omega_{j}+\kappa|t|}\left(\left|\eta_{j}^{+}\right|+\left|\eta_{j+1}^{-}\right|\right)+C e^{\kappa \omega_{j}+\kappa|t|}\left[\left|H\left(\omega_{j}\right)-H\left(-\omega_{j}\right)\right|\right] \\
& +C\left[e^{-\alpha t+\kappa|t|}\right]\left(\|\left(\mathcal{G}\left(V_{j}^{-}(\cdot), \cdot, c\right)\left\|_{\kappa}+\right\| \mathcal{G}\left(V_{j-1}^{+}(\cdot), \cdot c\right) \|_{\kappa}\right)\right. \\
& +C e^{-\alpha t+\kappa \omega_{j-1}+\kappa|t|}\left(\left|\eta_{j-1}^{+}\right|+\left|\eta_{j}^{-}\right|\right) .
\end{aligned}
$$

Hence, $e^{\kappa|t|}\left|\bar{V}_{j}(t)\right|_{\tilde{X}}$ can be bounded uniformly with respect to $t \in\left[0, \omega_{j}\right]$ and the choice of $\left\{\omega_{j}\right\}_{j \in \mathbb{Z}}$, if $2 \kappa<\gamma$. The case $0 \in\left[-\omega_{j-1}, 0\right]$ can be treated completely analogously.

With similar arguments one can now show that $l$ defines a contraction if $\rho>0$ (which appears in the definition of the cut-off function) is chosen small enough; again, this can be done uniformly with respect to the flight times. Claim B) is now true by the definition and construction of the functions $V_{j}^{+}, V_{j}^{-}$. Moreover, for fixed

$$
\left\{\omega_{j}\right\}_{j \in \mathbb{Z}} \in l^{\infty}\left(\mathbb{Z},\left(\omega_{*}, \infty\right)\right),
$$

the $C^{2}$-dependence on the values $\eta_{j}^{ \pm} \in \tilde{X}$ follows since $l$ depends bounded linearly on these values and hence its fixed points depend $C^{2}$ on $\left\{\eta_{j}^{ \pm}\right\}_{j \in \mathbb{Z} \text {. This }}$ shows D). Finally, let us show C). First of all note that if $\left\{\eta_{j}^{ \pm}\right\}_{j \in \mathbb{Z}}$ is fixed, then $l$ is a uniform contraction with respect to $\left\{\omega_{k}\right\}_{k \in \mathbb{Z}}$ in a small neighborhood of any prescribed $\left\{\omega_{k}^{*}\right\}_{k \in \mathbb{Z}} \in l^{\infty}\left(\mathbb{Z},\left(\omega_{*}, \infty\right)\right)$ if for all $k$ we have $\left|\omega_{k}^{*}\right|<C$ for some $C>0$. Then the claim $\mathrm{C}$ ) follows if we can show that $l$ depends continuously on the flight times; but this has been shown in [17]. Finally condition E) is also a consequence of the fact that $l$ depends $C^{2}$ on $c$; the property (20) of lemma 4 follows analogously to the hyperbolic case $m=0$, see for example lemma 11 in [5].

\section{Periodic travelling waves accompanying soli- tary waves in the FPU lattice}

In this section we want to use our results to discuss the existence of oscillatory solutions in the Fermi Pasta Ulam lattice

$$
\ddot{u}_{n}=\mathcal{V}^{\prime}\left(u_{n+1}-u_{n}\right)-\mathcal{V}^{\prime}\left(u_{n}-u_{n-1}\right), \quad n \in \mathbb{Z}
$$


for some potential $\mathcal{V}$ with $\mathcal{V}(0)=\mathcal{V}^{\prime}(0)=0$ and $\mathcal{V}^{\prime \prime}(0)=: \beta>0$. More precisely, setting $r_{n}=u_{n+1}-u_{n}$ we consider the equation

$$
\ddot{r}_{n}=\mathcal{V}^{\prime}\left(r_{n+1}\right)-2 \mathcal{V}^{\prime}\left(r_{n}\right)+\mathcal{V}^{\prime}\left(r_{n-1}\right), \quad n \in \mathbb{Z}
$$

and investigate the existence of periodic travelling waves near solitary wave solutions in this equation. Since our approach does in principal not rely on the fact that the solitary wave profile is small (i.e. close to the steady state 0 in some sense), we will set up the reduction method in the next section without assuming smallness of the initial solitary wave solution. Our main results of this section are summarized in theorem 4, which assures the existence of periodic travelling waves under suitable generic assumptions on the original solitary wave. We will afterward validate all hypotheses in section 6.2 for a specific case by making use of a result of Pego and Friesecke [8], who proved the existence of solitary waves and obtained very detailed informations concerning these solutions (see also theorem 5). As a main result, we show that each such wave is accompanied by a family of symmetric periodic travelling waves of (38) with arbitrary large period. Moreover, we show that these periodic waves persist under small reversible perturbations of the FPU-lattice and induce symmetric solitary waves for the perturbed equation, see section 6.3.

In the course of proving theorem 4, we will apply the reduction method introduced earlier in this article and thereby derive a lot of important properties concerning the jump function $\xi_{j}$ in the context of general Hamiltonian or timereversible LDEs. For example, if one studies the existence of periodic travelling then lemma 11 in connection with lemma 12 allows one to count and reduce all fixpoint-components of the underlying reversibility from the bifurcation equations, which helps to reduce the number of bifurcation equations.

\subsection{The reduction process}

In this section we obtain a general result concerning the existence of periodic travelling waves near solitary waves in time-reversible lattice differential equations, which possess conserved quantities. For the sake of illustration we will focus on the Fermi-Pasta-Ulam lattice differential equation though the reduction procedure holds true in more general equations. The main result is stated in section 6.1.5. It states that under generic conditions every supersonic solitary wave (i.e. whose wave speed $c_{*}$ satisfies $\mathcal{V}^{\prime \prime}(0)=\beta<c_{*}^{2}$ ) of the FPU lattice is accompanied by periodic travelling waves. Moreover, these periodic waves have the same wave speed as the solitary wave and their period can become arbitrary large.

Only when it comes to the validation of the required assumptions of our main result, we will make strongly use of results obtained by Pego and Friesecke [13] in the framework of the Fermi-Pasta-Ulam lattice. We start by making the next assumption.

\section{Hypothesis 3}

The equation (38) possesses a solitary wave $r_{n}(t)=\psi\left(n-c_{*} t\right)$ for some travelling wave speed $c_{*}^{2}>\beta$ (in particular the wave is supersonic). The function $\psi(\tau)$ decays exponentially to zero as $\tau \rightarrow \pm \infty$ and $\psi$ is an even function. 
Let us remark that any homoclinic solution in the case $c_{*}^{2}>\beta$ has to decay with exponential rate asymptotically. In fact, this is consequence of the dynamic on a center manifold which shows that any steady state on the center manifold can not be approached by a different solution on the center manifold in forward time. Hence, any homoclinic solution has to approach a steady state on the center manifold in forward time along a strong stable direction and hence with exponential rate (see also the proof of theorem 6 where these arguments have been stated more explicitly).

The existence of such solitary wave solutions as in 3 has been proved in [7] and [8] by using variational methods and renormalization techniques, respectively (for more details, see the next section). The assumptions concerning the evenness actually implies that the orbit of the solution is invariant with respect the transformation $\psi(\tau) \mapsto \psi(-\tau)$ and therefore can be expressed in terms of the reversibility of $(37)$, see also below. The solution $r_{n}(t)=\psi\left(n-c_{*} t\right)$ now defines a solution $\psi(\tau)$ of the travelling wave equation

$$
c_{*}^{2} x^{\prime \prime}(\tau)=\mathcal{V}^{\prime}(x(\tau+1))-2 \mathcal{V}^{\prime}(x(\tau))+\mathcal{V}^{\prime}(x(\tau-1)),
$$

for $\tau=n-c_{*}$. In fact, $\psi$ defines a homoclinic solution of (39) to the steady state zero. An important observation is that any solution $x(\tau)$ of the travelling wave equation induces again a solution $x(-\tau)$. Posing (39) in abstract form leads to a reversible equation

$$
\dot{U}(t)=\mathcal{F}\left(U(t), c_{*}\right)
$$

More explicitly, this equation reads

$$
\left(\begin{array}{c}
\partial_{t} x(t) \\
\partial_{t} y(t) \\
\partial_{t} \phi(t, \cdot)
\end{array}\right)=\left(\begin{array}{c}
y(t) \\
1 / c_{*}^{2}\left[\mathcal{V}^{\prime}\left(\phi^{1}(t, 1)\right)-2 \mathcal{V}^{\prime}\left(\phi^{1}(t, 0)\right)+\mathcal{V}^{\prime}\left(\phi^{1}(t,-1)\right)\right] \\
\partial_{\theta} \phi(t, \cdot)
\end{array}\right)
$$

where $\phi^{1}$ denotes the first component of $\phi=\left(\phi^{1}, \phi^{2}\right):[-1,1] \rightarrow \mathbb{R}^{2}$ and $(x, y, \phi) \in X=\left\{\left(x, y,\left(\phi^{1}, \phi^{2}\right)\right) \in \mathbb{R}^{2} \times H^{1}\left([-1,1], \mathbb{R}^{2}\right): \phi(0)=(x, y)\right\}$. Moreover, the reversibility is given by

$$
\mathcal{R}\left(x, y, \phi^{1}(\theta), \phi^{2}(\theta)\right) \mapsto\left(x,-y, \phi^{1}(-\theta),-\phi^{2}(-\theta)\right)
$$

for any $\left(x, \xi, \phi^{1}(\theta), \phi^{2}(\underset{\tilde{X}}{)}) \in Y:=\mathbb{R}^{2} \times L^{2}\left([-1,1], \mathbb{R}^{2}\right)\right.$. For completeness we also specify the space $\tilde{X}$ in this particular case, which is

$$
\tilde{X}=\left\{\left(x, y, \phi^{1}, \phi^{2}\right) \in \mathbb{R}^{2} \times C^{0}\left([-1,1], \mathbb{R}^{2}\right):(x, y)=\left(\phi^{1}(0), \phi^{2}(0)\right)\right\} .
$$

A special solution of (40) is given by the homoclinic solution

$$
H(t):=\left(\psi(t), \psi^{\prime}(t), \psi_{t}(\cdot), \psi_{t}^{\prime}(\cdot)\right)
$$

and we observe that $\left(\psi(0), \psi^{\prime}(0), \psi(\cdot), \psi^{\prime}(\cdot)\right) \in \operatorname{Fix}(\mathcal{R})$ on account of the evenness of $\psi$ (as before, the index " $t$ " in $\psi_{t}$ is not a derivative!). Hence, $H$ is a symmetric homoclinic solution to the steady state zero. 


\subsubsection{The spectrum of the linearization at the steady state}

Since we want to apply our reduction procedure to the abstract equation (40), we first have to analyse the critical eigenvalues of the linearization of $D \mathcal{F}(0)$, regarded as a densely defined map from $Y$ to $Y$ with domain $X$. Due to the invariance $x \mapsto x+\eta$ of (39) for any $\eta \in \mathbb{R}$ we always will encounter critical spectrum. More precisely, an element $\lambda=i \omega$ in the critical spectrum is characterized by the fact that it provides a zero of the characteristic equation

$$
c_{*}^{2} \lambda^{2}=\beta\left(e^{\lambda}-2+e^{-\lambda}\right) .
$$

We obtain this equation by directly computing the resolvent of (40) linearized at zero. Alternatively, we can linearize (39) at $x=0$ and make the ansatz $e^{\lambda t}$ for the linearized equation, see [14] and also section 3.2. We point out that (42) is not satisfied for $\lambda=i \omega, \omega \neq 0$, in the case under investigation $c_{*}^{2}>\beta$, see lemma 1 in [21]. Moreover, the eigenvalue $\lambda=0$ has algebraic multiplicity 2 , since differentiating the characteristic equation with respect to $\lambda$ gives

$$
2 c_{*}^{2} \lambda=\beta\left(e^{\lambda}-e^{-\lambda}\right)
$$

which is always true for $\lambda=0$ (verify again section 3.2 for technical background in this respect). Here, we have used that the order of $\lambda=0$ as a zero of (42) coincides with its algebraic multiplicity as an eigenvalue. As a result, $\lambda=0$ induces a two-dimensional center eigenspace of $D \mathcal{F}\left(0, c_{*}\right)$ which is the span of the vectors $e_{1}=(1,0,1,0)$ and $e_{2}=(0,1, \theta, 1) \in \tilde{X}$.

\section{Notation}

For later reference we will denote by $E^{s}, E^{u}$ the stable and unstable eigenspace with respect to the linear map $D \mathcal{F}\left(0, c_{*}\right): X \subset Y \rightarrow Y$. Moreover, we denote by $E^{c s}, E^{c u}$ the center stable and center unstable eigenspace, respectively. The projection associated to the decomposition $E^{s}+E^{c u}=Y$ with range $E^{s}$ we denote by $\pi^{s}$ and similarly $\pi^{u}$ is defined.

\subsubsection{Geometric relations}

In this section we collect some informations concerning how center stable and center unstable eigenspace $E_{+}^{c s}(0), E_{-}^{c u}(0)$, respectively, intersect. Let us first observe that the linearization of (38) along $H(t)$, namely the equation

$$
\dot{Z}(t)=D \mathcal{F}\left(H(t), c_{*}\right) Z(t),
$$

possesses solution operators on $\mathbb{R}_{+}$(once condition (13) is satisfied, which we will assume from now on) with solution operators $\Phi_{+}^{c s}(t, s), \Phi_{+}^{s}(t, s) t \geq s \geq 0$ and $\Phi_{+}^{c u}(t, s), \Phi_{+}^{u}(t, s) s \geq t \geq 0$. Similarly, there exist solution operators on $\mathbb{R}_{-}$which we denote by $\Phi_{-}^{c s}(t, s), \Phi_{-}^{s}(t, s) s \leq t \leq 0$ and $\Phi_{-}^{c u}(t, s), \Phi_{-}^{u}(t, s)$ $t \leq s \leq 0$. Moreover, we can choose these solution operators in such a way that they satisfy (28) and in particular $\tilde{E}^{c}(\omega)$ (defined in lemma 6) is $\mathcal{R}$-invariant if $\omega>0$ is large enough. As usual, the index " $u$ " refers to the strong unstable part (hence, these solution operators decay exponentially in the distance $|t-s|$ 
on their domain of definition) and the solution operators with the index "cu" refers to the center-unstable part. An analogous convention holds for the indices " $s "$ and " $c s "$.

Let us also point out that in our particular case the space $E_{+}^{s}(t):=\operatorname{Rg}\left(\Phi_{+}^{s}(t, t)\right)$ possesses a two-dimensional complement $E_{+}^{c}(t)$ in $E_{+}^{c s}(t):=\operatorname{Rg}\left(\Phi_{+}^{c s}(t, t)\right)$ for any $t \geq 0$. This is true because the center eigenspace of $D \mathcal{F}\left(0, c_{*}\right)$ is twodimensional (note also lemma 2). Moreover, the space $E_{-}^{u}(t):=\operatorname{Rg}\left(\Phi_{-}^{u}(t, t)\right)$ possesses a two-dimensional complement $E_{-}^{c}(t)$ in $E_{-}^{c u}(t)$, which is defined by $E_{-}^{c u}(t):=\operatorname{Rg}\left(\Phi_{-}^{c u}(t, t)\right)$ for any $t \leq 0$ (and a $\sim$ on these spaces will again refer to these space as subspaces of $\tilde{X})$.

\section{Hypothesis 4}

It is true that

$$
E_{+}^{s}(0) \cap E_{-}^{u}(0)=\operatorname{span}\left\langle\partial_{t} H(0)\right\rangle
$$

As a consequence we can prove the next lemma, which plays one of the key roles in our proof since it helps us to count the parts of $\operatorname{Fix}(\mathcal{R})$ and $\operatorname{Fix}(-\mathcal{R})$ which are complementary to $\tilde{E}_{+}^{s}(0)+\tilde{E}_{-}^{u}(0)$.

\section{Lemma 11}

There exists two vectors $U_{+}, V_{+} \in \operatorname{Fix}(\mathcal{R})$ and a vector $U_{-} \in \operatorname{Fix}(-\mathcal{R})$ such that

$$
\tilde{E}_{+}^{s}(0)+\tilde{E}_{-}^{u}(0)+\operatorname{span}\left\langle U_{+}, V_{+}, U_{-}\right\rangle=\tilde{X}
$$

\section{Proof}

First note that $\partial_{t} H(0) \in \operatorname{Fix}(-\mathcal{R})$ on account of the definition of $\mathcal{R}$ and the fact that $H$ is an even function. Let us now observe that $\tilde{E}_{ \pm}^{c}(0)$ is twodimensional for both choices "+" and "-". Hence, both spaces have to intersect at least in a one-dimensional space $\operatorname{span}\left\langle V_{*}\right\rangle$ since the codimension of $\tilde{E}_{+}^{s}(0)+\tilde{E}_{-}^{u}(0)$ is three in $\tilde{X}$ (we refer to [15] in order to see how to rigorously count codimensions). Since the space $\operatorname{span}\left\langle V_{*}\right\rangle \subset \tilde{E}_{+}^{c}(0) \cap \tilde{E}_{-}^{c}(0)$ is $\mathcal{R}$-invariant, one of the following cases occurs. Either

i) $\operatorname{span}\left\langle V_{*}\right\rangle \subset \operatorname{Fix}(\mathcal{R})$ or

ii) $\operatorname{span}\left\langle V_{*}\right\rangle \subset \operatorname{Fix}(-\mathcal{R})$.

Let us only consider case $i$ ), since one can argue in case $i i$ ) analogously. Note that in case $i) V_{*}$ now plays the role of $V_{+}$in the statement of the lemma. In order to prove the existence of $U_{+}$, we need to define the spaces $\tilde{E}_{+}^{c s,+}(0):=$ $\tilde{E}_{+}^{s}(0)+\left\langle V_{*}\right\rangle$ and $\tilde{E}_{-}^{c u}(0):=\tilde{E}_{-}^{u}(0)+\left\langle V_{*}\right\rangle$. Similarly, we define the spaces $\tilde{E}^{c s,+}:=\tilde{E}^{s}+\operatorname{span}\langle(1,0,1,0)\rangle$ and $\tilde{E}_{+}^{c u}:=\tilde{E}^{u}+\operatorname{span}\langle(1,0,1,0)\rangle$, respectively, where $(1,0,1,0)$ is the vector of the center eigenspace which lies in $\operatorname{Fix}(\mathcal{R})$ (note that also $(0,1, \theta, 1) \in \operatorname{Fix}(-\mathcal{R})$ lies in the center eigenspace). By assumption, we therefore conclude that

$$
\tilde{E}_{-}^{c u}(0)+\tilde{E}_{+}^{c s}(0)
$$


has codimension two in $\tilde{X}$. We now consider the map

$$
\begin{aligned}
\kappa_{0}^{+}: \tilde{E}_{+}^{c s,+}(0) & \rightarrow \operatorname{Fix}(\mathcal{R}) \cap \tilde{X} \\
\kappa_{0}^{+}: U & \mapsto \frac{1}{2}(U+\mathcal{R} U) .
\end{aligned}
$$

Let us now argue that $\kappa_{0}^{+}$is a Fredholm operator and compute its index. In fact, we have that $\operatorname{ker}\left(\kappa_{0}^{+}\right)=\operatorname{span}\left\langle\partial_{t} H(0)\right\rangle$ (note that this kernel would be two-dimensional in case ii) here), the range is closed and any element in $\operatorname{ker}\left(\left(\kappa_{0}^{+}\right)^{*}\right)$ is characterized by the fact that it lies in a suitable complement of (44); for a proof that $\operatorname{ker}\left(\left(\kappa_{0}^{+}\right)^{*}\right)$ is in fact finite-dimensional and hence $\operatorname{Rg}\left(\kappa_{0}^{+}\right)$ has finite codimension see [17]. Hence, $\kappa_{0}^{+}$is in fact a Fredholm operator and we want to show that its index is zero (this would then provide the existence of the element $U_{+}$in the statement of the lemma.) The results in [17] now also imply that $i\left(\kappa_{0}^{+}\right)=i\left(\kappa_{\infty}^{+}\right)$, where

$$
\begin{aligned}
& \kappa_{\infty}^{+}: \tilde{E}^{c s,+} \rightarrow \operatorname{Fix}(\mathcal{R}) \cap \tilde{X} \\
& \kappa_{\infty}^{+}: U \mapsto \frac{1}{2}(U+\mathcal{R} U) .
\end{aligned}
$$

On account of the definition of $\tilde{E}^{c s,+}$ and the fact that $\tilde{E}^{s} \cap \tilde{E}^{u}=\{0\}$ at the steady state, we readily see that $\operatorname{ker}\left(\kappa_{\infty}^{+}\right)=\{0\}$. To compute $\operatorname{ker}\left(\left(\kappa_{\infty}^{+}\right)^{*}\right)$ is a little trickier; in order to do that let us consider the map $\kappa_{\infty}^{+}$first as a map from the space $E^{c s,+}$ to $\operatorname{Fix}(\mathcal{R})$ (and hence with respect to the $Y$-norm). Then an elementary computation shows that if $U_{*} \in \operatorname{ker}\left(\left(\kappa_{\infty}^{+}\right)^{*}\right) \subset \operatorname{Fix}(\mathcal{R})$ then

$$
U_{*} \perp\left(E^{c u,+}+E^{c s,+}\right) \text {. }
$$

But there already exists a nontrivial element in $\operatorname{Fix}(-\mathcal{R})$ satisfying the condition (46), namely $(0,1, \theta, 1)$, and since $E^{c u,+}+E^{c s,+}$ has codimension one in $Y$, the condition (46) cannot be satisfied for a nontrivial element $U_{*} \in \operatorname{Fix}(\mathcal{R})$. Hence, $\operatorname{ker}\left(\left(\kappa_{\infty}^{+}\right)^{*}\right)=\{0\}$ and now one can show easily that the same holds for the original map $\kappa_{\infty}^{+}$in (45). Therefore $i\left(\kappa_{0}^{+}\right)=i\left(\kappa_{\infty}^{+}\right)=0$. This finally shows the existence of the element $U_{+}$in the upper lemma.

In order to prove the existence of the element $U_{-} \in \operatorname{Fix}(-\mathcal{R})$ in the statement of the lemma, let us consider the function

$$
\begin{aligned}
& \kappa_{0}^{-}: \tilde{E}_{+}^{c s,+}(0) \rightarrow \operatorname{Fix}(-\mathcal{R}) \cap \tilde{X} \\
& \kappa_{0}^{-}: U \mapsto \frac{1}{2}(U-\mathcal{R} U)
\end{aligned}
$$

where now $\operatorname{ker}\left(\kappa_{0}^{-}\right)=\operatorname{span}\left\langle V_{+}\right\rangle$by assumption $\left.i\right)$. In order to show that the Fredholm index of this map is zero, let us again restrict to the steady state and consider the operator

$$
\begin{array}{r}
\kappa_{\infty}^{-}: \tilde{E}^{c s,+} \rightarrow \operatorname{Fix}(-\mathcal{R}) \cap \tilde{X} \\
\kappa_{\infty}^{-}: U \mapsto \frac{1}{2}(U-\mathcal{R} U) .
\end{array}
$$

We readily verify that then $\operatorname{ker}\left(\kappa_{\infty}^{-}\right)=\langle(1,0,1,0)\rangle$ and $\operatorname{ker}\left(\left(\kappa_{\infty}^{-}\right)^{*}\right)=\langle(0,1, \theta, 1)\rangle$ (by arguing again in the space $Y$ first). Hence, $i\left(\kappa_{0}^{-}\right)=0$ and the existence of an element $U_{-}$as in the statement of the lemma follows in case $i$ ). However, we can argue analogously in case $i i$ ) and the lemma is proved. 


\subsubsection{Analysis of the zero-set of the jump functions}

Under the upper assumptions we can now set up our reduction process. More precisely we can choose a three-dimensional, $\mathcal{R}$-invariant complement $\tilde{K}$ to $\tilde{E}_{+}^{s}(0)+\tilde{E}_{-}^{u}(0)$ in the form

$$
\tilde{K}=\operatorname{span}\left\langle U_{+}, V_{+}, U_{-}\right\rangle,
$$

where these vectors are specified in the upper lemma.

\section{Remark}

The following observation will be important for section 6.2. If $H$ is sufficiently small we know that $\Phi_{+}^{c s}(0,0), \Phi_{+}^{s}(0,0)$ are close to the linear operators $\pi^{c s}$ and $\pi^{s}$, respectively, with respect to the $L(\tilde{X}, \tilde{X})$ or $L(Y, Y)$-norm (see [16]). Here, $\pi^{c s}, \pi^{s}$ denote the spectral projections of $D \mathcal{F}\left(0, c_{*}\right)$ with respect to the center stable and stable eigenspace, respectively. Hence, $U_{-}$accounts for the $\operatorname{Fix}(-\mathcal{R})$ component of the center-part of $\tilde{E}_{+}^{c}(0)$ and therefore is close to $(0,1, \theta, 1)$ if $H$ is small enough. Indeed, note that $(0,1, \theta, 1)$ induces the $\operatorname{Fix}(-\mathcal{R})$ component of the center-part of $\operatorname{span}\langle(1,0,1,0),(0,1, \theta, 1)\rangle$ at the steady state. If $H$ is sufficiently small, we are therefore free to choose $U_{-}:=(0,1, \theta, 1)$ or some vector $U_{-}$close to this vector with respect to the $\tilde{X}$-norm within the space $\operatorname{Fix}(-\mathcal{R})$ and the resulting space $\tilde{K}$ still provides an $\mathcal{R}$-invariant complement.

Let us now use theorem 3 and consider the jump function

$$
\xi:\left(\omega_{*}, \infty\right) \times B_{\delta}(0) \rightarrow \tilde{K},
$$

with $\xi(\omega, \eta):=\xi_{1}\left(\omega, \omega, c_{*}, \eta\right)$ where $\eta=\left(\eta_{1}, \eta_{2}\right) \in \mathbb{R}^{2}$ denotes the parameter with respect to the center choice $V_{+}, U_{-}$and $B_{\delta}(0)$ denotes a ball centered at zero. If we can now prove the existence of a zero $(\omega, \eta)$ of $\xi$, then we obtain the existence of a periodic solution of the equation (38) of period $2 \omega$ (all $U_{i}^{+}, U_{i}^{-}$ are identical to $U_{1}^{+}, U_{1}^{-}$, respectively). The next observation is important in this respect.

\section{Lemma 12}

Choose $m_{+}=m_{-}=1$ (where these numbers appear in the construction of the jump functions and the statement of theorem 3) and fix some $M, \gamma>0$. Then we can choose the parameters $\eta_{1}, \eta_{2} \in \mathbb{R}$ as in theorem 3, with $\left|\eta_{1}\right|+\left|\eta_{2}\right|<$ $M e^{-\gamma \omega}$, such that the jump functions satisfy $\xi(\omega, \eta) \in \operatorname{Fix}(-\mathcal{R})$ for any $(\omega, \eta)$ with $\eta=\left(\eta_{1}, 0\right)$.

\section{Proof}

Before we begin with the proof, let us recall that the spaces $\operatorname{Rg} \bar{P}^{m_{+}}(\omega)$ and $\operatorname{Rg} \bar{P}^{m_{-}}(-\omega)$ are defined in definition 4. By definition $\xi(\omega, \eta)=\bar{V}^{+}(0)-\bar{V}^{-}(0)$ for some suitable solutions $\bar{V}^{+}(\cdot), \bar{V}^{-}(\cdot)$ of

$$
\partial_{t} V(t)=D \mathcal{F}(H(t), c) V(t)+\mathcal{G}(t, V(t))
$$

on $[0, \omega)$ and $(-\omega, 0]$, respectively. We recall that $H(0) \in \operatorname{Fix}(\mathcal{R})$ and therefore $\mathcal{R} H(t)=H(-t)$. A short calculation now shows that $Z^{-}(t):=\mathcal{R} \bar{V}^{+}(-t)$ 
solves the equation (47) on $0 \geq t \geq-\omega$ and $Z^{-}(0)=\mathcal{R} \bar{V}^{+}(0)$. Moreover, by construction $\bar{V}^{+}(t)$ satisfies the equation

$$
\bar{V}^{+}(0)=\bar{V}^{s, 0}+\Phi_{+}^{c u}(0, \omega) \bar{\eta}^{+}+\int_{\omega}^{0} \Phi_{+}^{c u}(0, s) \mathcal{G}\left(s, \bar{V}^{+}(s)\right) d s
$$

for some $\bar{\eta}^{+}=\left(\bar{\eta}^{++}+\bar{\eta}^{c+}, \bar{\eta}^{p+}\right) \in \bar{E}_{+}^{c u}(\omega) \times \operatorname{Rg} \bar{P}^{m_{+}}(\omega)$, where $\bar{\eta}^{++} \in \tilde{E}_{+}^{u u}(\omega)$, $\bar{\eta}^{c+} \in \tilde{E}^{c}(\omega) \cap \bar{E}_{+}^{c u}(\omega)$ and $\bar{\eta}^{p+}$ accounts for the one-dimensional parameter direction. Moreover, our construction in proof of lemma 6 we can choose the vector $\bar{\eta}^{c+}$ in such a way that $\bar{\eta}^{c-}:=\mathcal{R} \bar{\eta}^{c+}$ satisfies

$$
\operatorname{span}\left\langle\bar{\eta}^{c-}, \bar{\eta}^{c+}\right\rangle=\tilde{E}^{c}(\omega) .
$$

In fact, this is possible since the $\mathcal{R}$-invariant space $\tilde{E}^{c}$ approaches the center eigenspace $\operatorname{span}\langle(1,0,1,0),(0,1, \theta, 1)\rangle$ of the steady state as $\omega \rightarrow \infty$ and $\operatorname{span}\langle(1,0,1,0),(0,1, \theta, 1)\rangle \cap \operatorname{Fix}(\mathcal{R})$ as well as $\operatorname{span}\langle(1,0,1,0),(0,1, \theta, 1)\rangle \cap$ $\operatorname{Fix}(-\mathcal{R})$ are both one-dimensional (note also the remark preceding this lemma). Hence, we can choose $\bar{\eta}^{c+} \notin \operatorname{Fix}(\mathcal{R})$ and $\bar{\eta}^{c+} \notin \operatorname{Fix}(-\mathcal{R})$ satisfying (49). Similarly, taking into account that $\operatorname{Rg} \bar{P}^{m_{+}}(\omega)=\left\langle\bar{\eta}^{p+}\right\rangle$ we can redefine $\bar{\eta}^{p+}$ if necessary and construct $\bar{P}^{m_{-}}(-\omega)$ satisfying the properties in the definition 4 in such way that

$$
\operatorname{Rg}\left(\bar{P}^{m_{-}}(-\omega)\right)=\left\langle\mathcal{R} \bar{\eta}^{p+}\right\rangle ; \quad\left\langle\mathcal{R} \bar{\eta}^{p+}\right\rangle+\left\langle\bar{\eta}^{p+}\right\rangle=\tilde{E}^{c}(\omega)
$$

Finally we can choose $\bar{\eta}^{-} \in \bar{E}_{-}^{c s}(-\omega)$ (which appears in the integral equation (48) of $\left.\bar{V}^{-}(\cdot)\right)$ in the form $\bar{\eta}^{-}=\left(\bar{\eta}^{--}+\bar{\eta}^{c-}, \bar{\eta}^{p-}\right)$, where here

$$
\bar{\eta}^{--} \in \operatorname{Rg}\left(\left.\Phi_{-}^{s}(-\omega,-\omega)\right|_{\tilde{X}}\right)=\mathcal{R}\left[\tilde{E}_{+}^{u}(\omega)\right],
$$

$\bar{\eta}^{c-} \in \tilde{E}^{c}(\omega) \cap \tilde{E}_{-}^{c s}(-\omega)$ is defined in (49) and $\bar{\eta}^{p-}$ accounts for the onedimensional parameter space $\operatorname{Rg} \bar{P}^{m_{-}}(-\omega)$. We now apply $\mathcal{R}$ to equation (48). Taking into account our special choice of dichotomies (see again lemma 6 and its proof) and choosing any $\bar{\eta}^{p+}$ we have

$$
\begin{aligned}
\mathcal{R} \Phi_{+}^{c u}(0, \omega)\left[\bar{\eta}^{++}+\bar{\eta}^{c+}+\bar{\eta}^{p+}\right] & =\Phi_{-}^{c s}(0,-\omega) \mathcal{R}\left[\bar{\eta}^{++}+\bar{\eta}^{c+}+\bar{\eta}^{p+}\right] \\
& =\Phi_{-}^{c s}(0,-\omega)\left[\hat{\eta}^{--}+\hat{\eta}^{c-}+\bar{\eta}^{p-}\right]
\end{aligned}
$$

with $\bar{\eta}^{p-}:=\mathcal{R} \bar{\eta}^{p+}$ and where $\hat{\eta}^{--}+\hat{\eta}^{c-} \in \tilde{E}_{-}^{c s}(-\omega)$. Since $\mathcal{R} \mathcal{G}\left(s, \bar{V}^{+}(s)\right)=$ $-\mathcal{G}\left(-s, \mathcal{R} \bar{V}^{+}(s)\right)$ we conclude that

$$
\begin{aligned}
\mathcal{R} \bar{V}^{+}(0) & =\mathcal{R} \bar{V}^{s, 0}+\mathcal{R} \Phi_{+}^{c u}(0, \omega) \bar{\eta}^{+}+\int_{\omega}^{0} \mathcal{R} \Phi_{+}^{c u}(0, s) \mathcal{G}\left(s, \bar{V}^{+}(s)\right) d s \\
& =\mathcal{R} \bar{V}^{s, 0}+\Phi_{+}^{c s}(0,-\omega)\left(\bar{\eta}^{--}+\bar{\eta}^{c-}\right)-\int_{\omega}^{0} \Phi_{+}^{c s}(0,-s) \mathcal{G}\left(-s, \mathcal{R} \bar{V}^{+}(s)\right) d s \\
& =\mathcal{R} \bar{V}^{s, 0}+\Phi_{+}^{c s}(0,-\omega)\left(\bar{\eta}^{--}+\bar{\eta}^{c-}\right)+\int_{-\omega}^{0} \Phi_{+}^{c s}(0, s) \mathcal{G}\left(s, \mathcal{R} \bar{V}^{+}(-s)\right) d s
\end{aligned}
$$

We therefore see that $U^{+}(t):=\bar{V}^{+}(t)+H(t)$ and $\tilde{U}^{-}(t):=H(t)+\mathcal{R} \bar{V}^{+}(-t)=$ $H(t)+Z^{-}(t)$ satisfy all the assumptions of theorem 3 for the one-parameter 
family $\left(\eta^{p+}, \eta^{p-}\right)=\left(\eta^{p+}, \mathcal{R} \eta^{p+}\right)$. Therefore, $\tilde{U}_{-}(t)$ coincides with $H(t)+\bar{V}^{u}(t)$ by uniqueness (note that the parameter part vanishes and this is the only spot where non uniqueness could enter the integral equation). In particular $\mathcal{R} \bar{V}^{+}(-t)=\bar{V}^{-}(t)$. Finally, this shows that

$$
\mathcal{R} \xi(\omega, \eta)=\mathcal{R} \bar{V}^{+}(0)-\mathcal{R} \bar{V}^{-}(0)=\bar{V}^{-}(0)-\bar{V}^{+}(0)=-\xi(\omega, \eta) .
$$

The lemma tells us that $\xi(\omega, \eta)$ actually only takes values in the one-dimensional space $\operatorname{span}\left\langle U_{-}\right\rangle \subset \operatorname{Fix}(-\mathcal{R})$. Hence, we remain with just one single scalar bifurcation equation which is the main consequence of the last result. We will now show that $\xi=0$ for any $\omega>\omega_{*}$ large enough. In order to do that, let us note that there exists a conserved quantity, i.e. a first integral $J$ of (40). In fact, for any $U=(x, y, \phi(\cdot), \psi(\cdot)) \in \tilde{X}$ we can define the function

$$
J(U)=\psi(0)-\frac{1}{c_{*}^{2}}\left(\int_{0}^{1} \mathcal{V}^{\prime}(\phi(\theta))-\mathcal{V}^{\prime}(\phi(\theta-1)) d \theta\right)
$$

This quantity of course also defines a first integral of the original equation and we can show easily that $J(x(t+\cdot), \dot{x}(t+\cdot))$ is constant along any solution of (39). We now want to assume that $H(0)$ is a regular point for $J$. More precisely, we make the following assumption.

\section{Hypothesis 5}

The derivative of $J$ in direction $U_{-}$is non-zero, i.e. $D J(H(0))\left[U_{-}\right] \neq 0$.

By continuity, we also find the existence of a neighborhood $\mathcal{U}$ of $H(0)$ with respect to $\tilde{X}$ such that

$$
D J(Z)\left[U_{-}\right] \neq 0
$$

for all $Z \in \mathcal{U}$. Finally, let us consider the value $J\left(\bar{V}^{+}+H(0)\right)-J\left(\bar{V}^{-}+H(0)\right)$. This quantity is certainly zero, since the solutions associated to $\bar{V}^{+}+H(0)$ and $\bar{V}^{-}+H(0)$ lie in the same level set of $J$. We also have that $\bar{V}^{+}-\bar{V}^{-}=\lambda U_{-}$ for some $\lambda \in \mathbb{R}$, since $\xi(\omega, \eta)=\bar{V}^{+}-\bar{V}^{-}$takes values in span $\left\langle U_{-}\right\rangle$. Moreover, if $\omega>>0$ is large enough we have

$$
\begin{aligned}
0 & =J\left(\bar{V}^{+}+H(0)\right)-J\left(\bar{V}^{-}+H(0)\right) \\
& =\lambda \cdot \int_{0}^{1} D J\left(\theta \bar{V}^{+}+H(0)+(1-\theta) \bar{V}^{-}\right)\left[U_{-}\right] d \theta
\end{aligned}
$$

which can only be true if $\lambda=0$ because

$$
\int_{0}^{1} D J\left(\theta \bar{V}^{+}+H(0)+(1-\theta) \bar{V}^{-}\right)\left[U_{-}\right] d \theta \neq 0 .
$$

Hence, $\lambda=0$ and therefore $\xi(\omega, \eta)=0$ for all $\omega>0$ large enough and we conclude the existence of periodic solutions of (38). 


\subsubsection{Dependence on the travelling wave speed}

Let us note that all arguments of the previous sections addressed the existence of periodic travelling waves with a fixed wave speed $c_{*}$, which is the speed of the original solitary wave. We now want to analyze the properties of the constructed family of periodic travelling waves with respect to the wave speed. Note that these waves are induced by the initial values $H(0)+\bar{V}^{+}(0)$, where $\bar{V}^{+}(0)=\bar{V}_{c}^{+}(0)$ actually depends $C^{2}$ on $c \approx c_{*}$ by construction of the jump functions.

Fixing some $\omega>\omega_{*}$ large enough and some $\eta \approx 0$, we can now also deduce that $\xi(\omega, \eta, c)=0$ even after slight variation of the wave speed $c$ near the original speed $c_{*}$. In fact, our last arguments, which showed that all three components of $\xi\left(\omega, \eta, c_{*}\right) \in \tilde{K}$ vanish, hold true also for $c \approx c_{*}$. Hence, since $\bar{V}_{c}^{+}(0)$ and therefore also the induced periodic solution on $[-\omega, \omega]$ depends $C^{1}$ on $c$, we actually have proved that the family of periodic travelling waves

$$
U(t)=H(t)+\left\{\begin{array}{cc}
\bar{V}_{c}^{+}(t) & \omega \geq t \geq 0 \\
\bar{V}_{c}^{-}(t) & -\omega \leq t \leq 0
\end{array}\right.
$$

depends $C^{1}$ on $c \approx c_{*}\left(\right.$ regarded as elements in $\left.C^{0}([-\omega, \omega], \tilde{X})\right)$.

\subsubsection{The main result}

Summarizing, we can now state the main result of this section.

\section{Theorem 4 (Periodic travelling waves)}

Fix some $\gamma, M_{*}>0$ and assume that the assumption concerning the existence of a solitary wave with wave speed $c$, see hypothesis 3 , and the hypotheses 4, 5 are satisfied. Then there exists a $\omega_{*}>0$ and a $\omega$-dependent parameter $\eta_{1} \in \mathbb{R}$ with $\left|\eta_{1}\right| \leq M_{*} e^{-\gamma \omega}$ such that

A) for all $\omega>\omega_{*}$ and all $c$ with $\left|c-c_{*}\right|$ small enough, there exists a $2 \omega$ periodic, symmetric solution $p=p^{\omega, c, \eta_{1}}$ of

$$
c^{2} x^{\prime \prime}(\tau)=\mathcal{V}^{\prime}(x(\tau+1))-2 \mathcal{V}^{\prime}(x(\tau))+\mathcal{V}^{\prime}(x(\tau-1))
$$

which induces a periodic travelling wave solution

$$
r_{n}(t)=p^{\omega, c, \eta_{1}}(n-c t)
$$

of (38). In particular, $p^{\omega, c, \eta_{1}}(\tau)=p^{\omega, c, \eta_{1}}(-\tau)$ for all $\tau>0$. The waves $p^{\omega, c}(\cdot) \in C^{0}([-\omega, \omega], \mathbb{R})$ depend $C^{1}$ on $c \approx c_{*}$. Moreover, it is true that

$$
\sup _{[-\omega, \omega]}\left|p^{\omega, c, \eta_{1}}(\tau)-\psi(\tau)\right| \rightarrow 0
$$

as $\omega \rightarrow \infty$.

B) All reversible, small finite range perturbations of the Fermi-Pasta-Ulam lattice equation, that additionally admit a first integral, also possess a family of symmetric periodic travelling waves: Consider the equation

$$
\ddot{r}_{n}=\mathcal{V}^{\prime}\left(r_{n+1}\right)-2 \mathcal{V}^{\prime}\left(r_{n}\right)+\mathcal{V}^{\prime}\left(r_{n-1}\right)+\delta G\left(r_{n-M}, \ldots, r_{n}, \ldots, r_{n+M}\right),
$$


where for $M \in \mathbb{N}$ the function $G: \mathbb{R}^{2 M+1} \rightarrow \mathbb{R}$ is $C^{2}$ with $G(0)=0$. Suppose that $r_{n}(t)$ is a solution of (53) exactly if $r_{n}(-t)$ is a solution. Moreover, assume that the first integral $J$ defined in (51) admits a $C^{1}$ extension to a first integral $J^{\delta}$ of the associated travelling wave equation of (53) such that $D J^{\delta}(U)\left[U_{-}\right]$exists and depends continuously on $U \in \tilde{X}$ and $\delta \approx 0$ (where $U_{-}$has been defined in lemma 11). Then for $\delta \approx 0$ small enough and $\omega>0$ large enough, equation (53) possesses a $2 \omega$ periodic travelling waves $r_{n}^{\delta}(t)=p^{\omega, c, \eta_{1}, \delta}(n-c t)$ which is symmetric and differentiable with respect to $c$.

\section{Remark}

For example, a perturbation $G$ for which all assumptions listed in $B$ ) are satisfied is $G\left(r_{n-2}, r_{n}, r_{n+2}\right):=\tilde{G}\left(r_{n-2}\right)-2 \tilde{G}\left(r_{n}\right)+\tilde{G}\left(r_{n+2}\right)$ for some $C^{2}$-function $\tilde{G}$. In this case we can add the term

$$
1 / c_{*}^{2} \cdot \delta \cdot\left(\int_{0}^{2} W^{\prime}(r(\theta))-W^{\prime}(r(\theta-2)) d \theta\right)
$$

to $J(U)$ in order to obtain a first integral $J^{\delta}(U)$ for the perturbed travelling wave equation (here for the special case $c=c_{*}$ ).

\section{Proof of the theorem}

The proof of part $A$ ) follows from the results of the previous section. Let us only note that the symmetry property $p^{\omega, c}(\tau)=p^{\omega, c}(-\tau)$ of the periodic travelling waves follows by uniqueness of the solutions with respect to the Lyapunov-Schmidt reduction. In fact, if the orbit of $p$ would not be invariant under $\mathcal{R}$, we would get another solution of $\xi\left(\omega, c, \eta_{1}\right)=0$, see also the proof of lemma 12.

The proof of case $B$ ) also follows from the results obtained before: Let us observe that for $|\delta|$ small enough, the term $\delta G$ just amounts to a small bounded, reversible perturbation term in the integrand (50). Hence, going through the proof of lemma 12 the arguments do not change and therefore the bifurcation function $\xi=\xi\left(\omega, c, \eta_{1}, \delta\right)$ only takes values in the one-dimensional space $\operatorname{Fix}(-\mathcal{R}) \cap \tilde{K}$. Now using the first integral $J^{\delta}$ shows that in fact $\xi=0$ which ends the proof.

Let us also stress that we have so far not really used the fact that the twodimensional center eigenspace of $D \mathcal{F}(0, c)$ stems from an eigenvalue zero of algebraic multiplicity two. In different reversible lattice differential equations (where the existence of a zero eigenvalue is not a priori forced as in the FPU lattice) we can therefore prove theorem 4 completely analogous. For example, a typical alternative would be the existence of two simple purely imaginary eigenvalues $\pm i \omega$, which are present in the Klein Gordon lattice for an open set of parameters, for example. The occurrence of such eigenvalues now generically forces the existence of a family of small periodic solutions near the steady state by the Lyapunov Center theorem [16], which lie on and actually define the center manifold (note that center manifolds in this context have been proved rigorously, see $[20,21,14])$. As a consequence, any homoclinic solution has 
to approach the steady state along a strong direction as $t \rightarrow \pm \infty$; hence with exponential rate. This shows that the condition on exponential decay of the homoclinic solution, which was used in our approach, is often satisfied automatically in some cases.

\subsection{An explicit example: Periodic solutions near small solitary waves}

In this section we want to consider an explicit scenario, where all the hypotheses of theorem 3 section can be validated. As a starting point, we state a result obtained by Pego and Friesecke (stated for our purposes).

\section{Theorem 5 (Existence of solitary waves)}

Assume that $\mathcal{V} \in C^{4}$ with $\mathcal{V}(0)=\mathcal{V}^{\prime}(0)=0$ and $\mathcal{V}^{\prime \prime}(0)=: \beta>0$ and $\mathcal{V}^{\prime \prime \prime}(0)=$ : $\gamma \neq 0$. Then if $c^{2}$ is sufficiently close to $c_{*}^{2}=\beta$ the following is true:

a) Equation (39) admits an up-to-translation unique single-pulse solution with wave speed $c$ and with values in an arbitrary interval, containing zero, on which $\mathcal{V}^{\prime}(r)>r$. More precisely, the solution is unique in $H^{1}(\mathbb{R}, \mathbb{R})$ with the property that its derivative vanishes only at one point.

b) Denote by $\psi=\psi^{c}$ the profile with critical point at $x=0$ and define a parameter $\varepsilon$ via $c / c_{*}=1+\varepsilon^{2} / 24$. Then $\psi^{c}$ is even and positive in the case that $\mathcal{V}^{\prime \prime \prime}(0)>0$, has a characteristic width of order $\frac{1}{\varepsilon}$ and height of order $\varepsilon^{2}$. Moreover,

$$
\left\|\frac{1}{\varepsilon^{2}} \psi^{c}\left(\frac{\dot{y}}{\varepsilon}\right)-\phi_{1}\right\|_{H^{1}} \leq C \varepsilon^{2},
$$

where $C>0$ does not depend on $\varepsilon$ and $\phi_{1}$ is non-zero and solves the $K d V$ travelling wave equation $-\phi+\phi_{x x}+12(\gamma / \beta) \phi^{2} / 2=0$, i.e.

$$
\phi_{1}(x)=\frac{\beta}{\gamma}(1 / 2 \cdot \operatorname{sech}(1 / 2 x))^{2} .
$$

Let us now set $H(t)=H^{\varepsilon}(t)=\left(\psi^{c}(t), \psi_{t}^{c}\right)$ where $c=c(\varepsilon)$. We can now validate the hypotheses necessary for theorem 4 .

\section{Lemma 13}

Hypothesis 4 is satisfied if $\varepsilon>0$ (and therefore also $\left\|\psi^{c}(\cdot)\right\|_{\infty}$ ) is small enough.

\section{Proof}

Note first of all that the hypothesis (13) is satisfied if $\varepsilon>0$ is small enough, since the variational equation along $\psi^{c}$ reads

$$
c^{2} \cdot \ddot{y}(t)=\mathcal{V}^{\prime \prime}\left(\psi^{c}(t+1)\right) y(t+1)-2 \mathcal{V}^{\prime \prime}\left(\psi^{c}(t)\right) y(t)+\mathcal{V}^{\prime \prime}\left(\psi^{c}(t-1)\right) y(t-1)
$$

and $\mathcal{V}^{\prime \prime}\left(\psi^{c}(t)\right) \approx \beta \neq 0$ for any point $t \in \mathbb{R}$, so that hypothesis (13) is true (and in particular the existence of trichotomies for the abstract variational equation is granted). The proof that $E_{+}^{s}(0) \cap E_{-}^{u}(0)$ is only one-dimensional 
follows from [9] (see the paragraph before theorem 1.2 there) and we omit it.

We will now show that all assumptions of theorem 4 are satisfied. In particular, we prove the existence of a family of periodic travelling waves $p=p^{\omega}$ of arbitrary large period near the solitary wave.

\section{Lemma 14}

Hypothesis 5 is satisfied if $\varepsilon>0$ is small enough.

\section{Proof}

For $\varepsilon$ small enough (and therefore $H$ small) we have that $c^{2} \approx \mathcal{V}^{\prime \prime}(0)$ but $c^{2}>\mathcal{V}^{\prime \prime}(0)$. Let us now consider

$$
U_{-}^{\delta}:=\left(0,1, \theta+\delta \theta^{3}, 1+\delta 3 \theta^{3}\right)
$$

for some $\delta>0$ small. Let us in particular choose $\delta$ small enough such that $U_{-}^{\delta}$ still induces a complement (see also the remark of the previous section). This certainly works since $U_{-}^{\delta} \approx(0,1, \theta, 1)$, which spans $\tilde{E}^{c} \cap \operatorname{Fix}(-\mathcal{R})$, where $\tilde{E}^{c} \subset \tilde{X}$ denotes the generalized center eigenspace of $D \mathcal{F}(0, c)$. Let us now note that for any vector $V=(\tau, \eta, \phi(\cdot), \psi(\cdot))$ we have

$$
D J(H(0))[V]=\psi(0)-\frac{1}{c^{2}}\left(\int_{0}^{1} \mathcal{V}^{\prime}(0) \phi(\theta)-\mathcal{V}^{\prime \prime}(0) \psi(\theta-1) d \theta\right) .
$$

In particular for $V=U_{-}^{\delta}$ we obtain

$$
\begin{aligned}
D J(0)\left[U_{-}^{\delta}\right] & =1-\frac{1}{c^{2}}\left(\int_{0}^{1} \mathcal{V}^{\prime \prime}(0)\left[\theta+\delta \theta^{3}\right]-\mathcal{V}^{\prime \prime}(0)\left[(\theta-1)+\delta(\theta-1)^{3}\right] d \theta\right) \\
& =1-\frac{1}{c^{2}} \mathcal{V}^{\prime \prime}(0)\left(\int_{0}^{1} 1+3 \theta^{2} \delta+\delta-3 \theta \delta d \theta\right) \\
& =1-\frac{1}{c^{2}} \mathcal{V}^{\prime \prime}(0)-\mathcal{V}^{\prime \prime}(0) \frac{1}{c^{2}}\left(\left[\theta^{3} \delta\right]_{0}^{1}+\delta-\left[\frac{3}{2} \theta^{2} \delta\right]_{0}^{1}\right) \\
& =\left(1-\frac{1}{c^{2}} \mathcal{V}^{\prime \prime}(0)\right)-\left(\frac{1}{c^{2}} \beta \frac{\delta}{2}\right) .
\end{aligned}
$$

If now $\delta$ is chosen small and afterward $\varepsilon=\varepsilon(\delta)$ is chosen sufficiently small, then $D J\left(H^{\varepsilon}\right)\left[U_{-}^{\delta}\right] \approx D J(0)\left[U_{-}^{\delta}\right]$. Moreover, the first bracket in the last equation of $D J(0)\left[U_{-}^{\delta}\right]$ approaches zero and the second bracket approaches the value $\delta / 2$ since $\beta / c^{2} \approx 1$. This finally shows that

$$
\operatorname{DJ}\left(H^{\varepsilon}(0)\right)\left[U_{-}^{\delta}\right] \neq 0,
$$

if $\delta$ is chosen small and afterward $\varepsilon=\varepsilon(\delta)$ is chosen sufficiently small which proves the lemma.

\subsection{Solitary waves in reversible lattices close to the FPU lattice}

The theorem 4 implies that the family of periodic travelling waves in part A) persists after small reversible perturbations of the FPU-lattice and induces a 
family $r_{n}^{\delta}(t)=p^{\omega, c, \eta_{1}, \delta}(n-c t)$ for $\delta \approx 0$. Moreover, for fixed $c \approx c_{*}$ and $\omega \rightarrow 0$ the period of the spatial profile $p^{\omega, c, \eta_{1}, \delta}(\cdot)$ tends to infinity. It is therefore natural to ask, whether there exists a solitary $\tilde{r}_{n}(t)=\tilde{\psi}(n-c t)$ wave which is induced by these waves in the sense of (52). That this is indeed the case is the content of the next theorem.

\section{Theorem 6}

Assume that the assumptions of theorem 4 are satisfied and consider a perturbation of the FPU-lattice in the sense of case B) in theorem 4, such that the characteristic equation at zero with respect to the travelling wave equation of (53) either possesses $\lambda=0$ as a solution or the zeros which are closest to 0 are real with nontrivial real part. Then for every $c$ sufficiently close to $c_{*}$ and any $\delta>0$ sufficiently small, there exists a solitary wave solution $\tilde{r}_{n}(t)=\tilde{\psi}(n-c t)$ of (53), such that for any $[-C, C], C>0$, we have

$$
\sup _{[-C, C]}\left|p^{\omega, c, \eta_{1}, \delta}(\tau)-\tilde{\psi}(\tau)\right| \rightarrow 0
$$

as $\omega \rightarrow \infty$.

\section{Proof}

For the sake of brevity let us only give a sketch of the proof. Let us set $\eta_{1}=0$ for simplicity. Then we have that for any $\varepsilon_{*}>0$ small enough and $c \approx c_{*}$ there are $\omega_{*}>0, \delta>0$ small such that

$$
\left|p^{\omega, c, 0, \delta}(\tau)-\psi(\tau)\right|<\varepsilon_{*}
$$

for $\omega>\omega_{*}$ (where $\psi$ denotes the solitary wave profile for the case $\delta=0$ ). Moreover, as $\omega \rightarrow \infty$ we can choose a subsequence of the periodic waves $p^{\omega, c, 0, \delta}$ on any compact interval $[-C, C], C>0$, which converges uniformly to some bounded even function $\tilde{\psi}: \mathbb{R} \rightarrow \mathbb{R}$ which again solves the travelling wave equation associated to (53). Using (55) we can see that $\tilde{\psi}$ and therefore also $\tilde{H}(t):=\left(\tilde{\psi}(t), \tilde{\psi}_{t}\right)$, which solves the abstract equation $\dot{U}=\mathcal{F}(U, c, \delta)$ associated to the travelling wave equation of (53), stays in a $\gamma$-neighborhood of zero for all $t>0$ large enough, where $\gamma \rightarrow 0$ as $\varepsilon_{*} \rightarrow 0$. Hence, $\tilde{H}(t)$ lies in the local center stable manifold $W^{c s}(0)$ of zero for $t>0$ large enough. We recall that the existence of $W^{c s}(0) \subset \tilde{X}$ has been proved in [16]. This manifold has the property that it contains every initial value in $\tilde{X}$ sufficiently close to zero, which induces a solution $U(t)$ that exists for all forward times $t>0$ and stays within a small neighborhood of zero for all $t>0$. Moreover, any such solution approaches an orbit on the center manifold asymptotically in forward time $t \rightarrow \infty$, see [16]. From this it already follows that $\tilde{H}$ has to approach a symmetric steady state in forward time, since any other solution on the center manifold leaves a $\gamma$-neighborhood of zero in finite time if $\gamma>0$ is chosen small enough. Let us show this last claim in a little more detail, where properties of center manifolds with respect to the equation $\dot{U}=\mathcal{F}(U, c, \delta)$, are collected in [16]. In fact, without even computing the Taylor expansion of the reduced equation on the center manifold, we a priori know several facts: The reduced equation on the center manifold is two dimensional with zero being an equilibrium which can be seen immediately by taking into account the special 
form of the perturbation in (53). Moreover, the reduced equation on the center manifold is reversible with respect to the reverser $\mathcal{R}$ (see (41)), which follows from the explicit choice of our perturbation in (53) together with the properties of any appropriate center manifold which respects the reversibility, see [16]. If, we consider the eigenvalues of the linearization at zero, two different scenarios can occur by assumption: a) the eigenvalues are real and non-zero; b) zero is a double eigenvalue which is geometrically simple. We only consider b), since case a) is easy to handle: In fact, in case the steady state zero with respect to the equation $\dot{U}=\mathcal{F}(U, c, \delta)$ possesses a strong stable and strong unstable manifold (in particular with trivial center direction) and therefore the solution $\tilde{H}$ has to approach the steady state 0 as $t \rightarrow \infty$ is therefore a symmetric homoclinic solution. Let us therefore assume that case $b$ ) is true; then the linearization at zero possesses an $\mathcal{R}$-invariant, two-dimensional center eigenspace which is the span of a component in $\operatorname{Fix}(\mathcal{R})$ and one component in $\operatorname{Fix}(-\mathcal{R})$. We can therefore write down the Taylor expansion of the reduced vector field on the center manifold, which reads

$$
\left(\begin{array}{l}
\dot{x} \\
\dot{y}
\end{array}\right)=\left(\begin{array}{c}
y+b x y+h . o . t . \\
h y^{2}+g x^{2}+\text { h.o.t. }
\end{array}\right)
$$

for some $b, g, h \in \mathbb{R}$, where $g=0$ for $\delta=0$ and h.o.t. denote higher order terms which are at least cubic in $x, y$. Moreover, the variable $x$ denotes the $\operatorname{Fix}(\mathcal{R})$-component and $y$ the $\operatorname{Fix}(-\mathcal{R})$-component of the reduced vector field. Note that (56) holds also true in the case of our original FPU lattice, i.e. in the case $\delta=0$ (where $g=0$ on account of the line of symmetric steady states). One can now easily check that every solution of (56) which is not a steady state leaves any sufficiently small neighborhood of zero in forward time. However, since $\tilde{H}(t)$ stays in sufficiently small neighborhood of zero for all $t>0$ large enough we conclude that $\tilde{H}(t)$ approaches a symmetric steady state, possibly different from zero, as $t \rightarrow \infty$. Hence, since $\tilde{H}$ is an even function we conclude that $\tilde{H}$ is a symmetric homoclinic solution and therefore defines a solitary wave solution of (53) which by construction is approached by the profiles of the periodic waves.

The construction of these solitary wave solutions for the case $\delta \approx 0$ actually shows that the associated profile $\tilde{\psi}$ must be close to the solitary wave profile $\psi$ for the case $\delta=0$ with respect to the supremum norm. Moreover, if the solitary wave with respect to $\psi$ is actually linearly stable (see $[8,9,10,11]$ for a definition of stability in this context) we therefore expect the perturbed solitary waves to be linearly stable as well, where methods based on the results of [12] and [34, 35] should be applicable. In the previous section we have shown that all assumptions of theorem 4 can be verified for a special family of solitary waves constructed by Pego and Friesecke $[8,9,10,11]$, where the authors have actually proved that the solitary waves are in fact non-linearly stable with respect to the dynamics of the FPU-lattice. In particular, the waves are linearly stable and we therefore expect the solitary waves for the slightly perturbed FPU-lattice to be linearly stable as well. 


\section{References}

[1] A. Chmaj, X. Ren Homoclinic Solutions of an Integral Equation: Existence and Stability, J. Diff. Eq. 155, pp. 17 - 43, 1999

[2] S.N. Chow, X.B. Lin Bifurcation of homoclinic orbits asymptotic to saddle-node equilibrium, J. Diff. and Integr. Eqs. 3, pp. 435 - 466, 1990

[3] R. Devaney Blue sky catastrophes in reversible and Hamiltonian systems, Ind. Univ. Math. J. 26, pp. 247 - 263, 1977.

[4] O. Diekman, S.V. Lunel, S.A. van Gils, H.O. Walther Delay equations, Springer Verlag, 1994

[5] B. Fiedler, A. Vanderbauwhede Homoclinic period blow-up in reversible and conservative systems, Z. angew. Math. Phys. 43, pp. 293-318, 1992.

[6] E. Fermi, J. Pasta, S. Ulam Studies of nonlinear problems, Los Alamos Sc. Rep. LA-1940, 1995.

[7] G. Friesecke, J. A. D. Wattis Existence Theorem for Solitary Waves on Lattices, Comm. Math. Phys. 161, pp. $391-418,1994$

[8] G. Friesecke, R. L. Pego Solitary waves on FPU lattices I: Qualitative properties, renormalization and continuum limit, Nonlin. 12, pp. 1601 1627, 1999

[9] G. Friesecke, R. L. Pego Solitary waves on FPU lattices II: Linear implies nonlinear stability, Nonlin. 15, pp. 1601 - 1627, 2002, pp. $1344-1359$

[10] G. Friesecke, R. L. Pego Solitary waves on FPU lattices III: Howlandtypw Floquet theory, Nonlin. 17, pp. 207 - 227, 2004

[11] G. Friesecke, R. L. Pego Solitary waves on FPU lattices IV: Proof of stability at low energy, Nonlin. 17, pp. 229 - 251, 2004

[12] S. Benzoni-Gavage, P. Huot, F. Rousset Nonlinear stability of semidiscret shock waves, SIAM J. math. analy. 35, no. 3, pp. 639 - 7072003

[13] M. Georgi Exponential Dichotomies and Transversal Homoclinic Points in Functional Differential Equations of Mixed Type, J. of Diff. Eq., submitted, 2007

[14] M. Georgi Interaction of Homoclinic Solutions and Hopf Points in Functional Differential Equations of Mixed Type, Proc. Roy. Soc. Eding., accepted

[15] M. Georgi Structural Stability of Travelling Waves in an Integrodifferential Equation, Dyn. Sys. 23, no. 1, pp. 109 - 135, 2008

[16] M. Georgi Bifurcations of homoclinic orbits to non-hyperbolic equilibria in reversible lattice differential equations, Nonlin. 21 no. 4, pp. 735 - 763, 2008 
[17] M. Georgi, A. Jukes, J. Lamb, K. Webster Homoclinic period blow up and the detection of multipulse solutions in lattice differential equations, Preprint, 2008

[18] J. Härterich, B. Sandstede, A. Scheel Exponential dichotomies for linear non-autonomous functional differential equations of mixed type, Ind. Univ. Math. J. 51, pp. $1081-1109,2002$

[19] H.J. Hupkes, S.M. Verduyn Lunel, Lin's Method and Homoclinic Bifurcations for Functional Differential Equations of Mixed Type, preprint, 2008

[20] G. Iooss, K. Kirchgässner Travelling Waves in a Chain of Coupled Nonlinear Oscillators, Comm. Math. Phys. 211, pp. 439 - 262, 2000

[21] G. Iooss, K. Kirchgässner Travelling Waves in the Fermi-Pasta-Ulam lattice, Nonlin. 13, pp. 849 - 866, 2000

[22] A. Jukes, On homoclinic bifurcations with symmetry, Dissertation Thesis, Imp. Coll., London, 2006

[23] J. Knobloch, Lin's method for discrete and continuous dynamical systems and applications, Habilitation thesis, TU-Ilmenau, 2004

[24] X.B. Lin, Using Melnikovs Method to solve Shilnikovs problem, Proc. of Roy. Soc. Eding., 116 A, pp. 295 - 325, 1990

[25] X.B. Lin, Shadowing lemma and singularly perturbed boundary value problems, SIAM J. Appl. Math 49, pp. 26 - 54, 1989

[26] X.B. Lin, Homoclinic bifurcations with weakly expnading center manifolds, Dynamics Reported 5, Expos. in Dyn. Sys., C. Jones, U. Kirchgraber, H.O. Walther (eds.), pp. $99-189,1996$

[27] S.V. Lunel, J. Mallet-Paret Exponential dichotomies and Wiener-Hopf factorizations for mixed-type functional dierential equations, preprint, 2001

[28] D. Peterhof, B. Sandstede, A. Scheel Exponential dichotomies for solitarywave solutions of semilinear elliptic equations on infinite cylinders, J. Diff. Eq. 140, pp. $266-308,1997$

[29] J. Mallet-Paret The Fredholm Alternative for functional differential equations of mixed type, J. Dyn. and Diff. Eq. 11, pp. 1 - 47, 1997

[30] J. Mallet-Paret Crystallographic Pinning: Direction Dependent Pinning in Lattice Differential Equations, J. Diff. Eq., to appear

[31] A. Pankov Travelling waves and periodic oscillations in Fermi-PastaUlam lattices, Imp. Coll. Press, World Sc. Publ., Singapore, 2005

[32] A. Rustichini Functional differential equations of mixed type: the linear autonomous case, J. Dyn. and Diff. Eq. 11, pp. 121 - 143, 1987. 
[33] B. Sandstede Verzweigungstheorie homokliner Verdopplungen, Ph.D. thesis, University of Stuttgart, 1993

[34] B. Sandstede, A. Scheel On the Structure of Spectra of Modulated Travelling Waves, Math. Nachrichten 232, pp. 39 - 93, 1999

[35] B. Sandstede, A. Scheel Essential Instabilities of Pulses and Bifurcations to Modulated Travelling Waves, Proc. of Roy. Soc. of Edin. 129 A, pp. 1263 - 1290, 1999

[36] B. Sandstede, A. Scheel Essential Instabilities of fronts: bifurcations, and bifurcation failure, Dyn. Sys. 16, pp. 1 - 28, 2001 